\title{
GLOBAL SOLUTIONS TO THE OLDROYD-B MODEL WITH A CLASS OF LARGE INITIAL DATA
}

\author{
DAOYUAN FANG, RUIZHAO ZI
}

\begin{abstract}
Consider a global wellposed problem for the incompressible Oldroyd-B model. It is shown that this set of equations admits a unique global solution provided the initial horizontal velocity $u_{0}^{h}$, the product $\omega u_{0}^{d}$ of the coupling parameter $\omega$ and initial the vertical velocity $u_{0}^{d}$, and initial symmetric tensor of constrains $\tau_{0}$ are sufficient small in the scaling invariant Besov space $\dot{B}_{2,1}^{\frac{d}{2}-1} \times \dot{B}_{2,1}^{\frac{d}{2}}, d \geq 2$. In particular, the result implies the global well-posedness of Oldroyd-B model with large initial vertical velocity $u_{0}^{d}$.
\end{abstract}

\section{INTRODUCTION}

In this paper, we focus on a prototypical model for viscoelastic fluids, the so called Oldroyd-B model, which was originally introduced by J.G. Oldroyd [28]. This type of fluids is described by the following set of equations

$$
\left\{\begin{array}{l}
\operatorname{Re}\left(u_{t}+(u \cdot \nabla) u\right)-(1-\omega) \Delta u+\nabla \Pi=\operatorname{div} \tau, \\
\operatorname{We}\left(\tau_{t}+(u \cdot \nabla) \tau+g_{\alpha}(\tau, \nabla u)\right)+\tau=2 \omega D(u), \\
\operatorname{div} u=0,
\end{array}\right.
$$

where $u$ is the velocity of the fluid, $\tau$ is the symmetric tensor of constrains, and $\Pi$ is the pressure which can be regarded as the Lagrange multiplier for the divergence free condition. Re and We denote the Reynolds and Weissenberg number of the fluid, respectively. $\omega \in(0,1)$ is the coupling parameter. Moreover, the quadratic term $g_{\alpha}$ is given by $g_{\alpha}(\tau, \nabla u):=\tau W(u)-W(u) \tau-\alpha(D(u) \tau+\tau D(u))$ for some $\alpha \in[-1,1]$, where $D(u)=1 / 2\left(\nabla u+(\nabla u)^{\top}\right)$ is the deformation tensor and $W(u)=1 / 2\left(\nabla u-(\nabla u)^{\top}\right)$ is the vorticity tensor.

A brief derivation of system (1.1) is as follows. In fact, the dynamics of homogeneous, isothermal, and incompressible fluid is described by

$$
\left\{\begin{aligned}
\partial_{t} u+(u \cdot \nabla) u & =\operatorname{div} \mathbb{T} \\
\operatorname{div} u & =0
\end{aligned}\right.
$$

where $u, \mathbb{T}$ are the velocity and stress tensor, respectively. Moreover, $\mathbb{T}$ can be decomposed into $-p$ Id $+\tau$, where $p$ denotes the pressure of the fluid and $\tau$ the tangential part of the stress tensor, respectively. For the Oldroyd-B model, $\tau$ is given by the relation

$$
\tau+\lambda \frac{\mathcal{D}_{\alpha} \tau}{\mathcal{D} t}=2 \eta\left(D(u)+\mu \frac{\mathcal{D}_{\alpha} D(u)}{\mathcal{D} t}\right)
$$

where $\frac{\mathcal{D}_{\alpha}}{\mathcal{D} t}$ denotes the "objective derivative"

$$
\frac{\mathcal{D}_{\alpha} \tau}{\mathcal{D} t}=\partial_{t} \tau+(u \cdot \nabla) \tau+g_{\alpha}(\tau, \nabla u)
$$

The parameters $\lambda>\mu>0$ denote the relaxation and retardation time, respectively, and $\eta$ is the viscosity of the fluid.

2010 Mathematics Subject Classification. 76A10, 76D03.

Key words and phrases. Oldroyd-B model, critical Besov space, global existence, large data. 
The tangential part of the stress tensor $\tau$ can be decomposed as $\tau=\tau_{N}+\tau_{e}$ where $\tau_{N}=2 \eta \frac{\mu}{\lambda} D(u)$ corresponds to the Newtonian part and $\tau_{e}$ to the purely elastic part. Denoting $\tau_{e}=\tau$, the above equations can be rewritten as

$$
\left\{\begin{aligned}
\partial_{t} u+(u \cdot \nabla) u-\eta(1-\omega) \Delta u+\nabla p & =\operatorname{div} \tau \\
\operatorname{div} u & =0 \\
\lambda\left(\partial_{t} \tau+(u \cdot \nabla) \tau+g_{\alpha}(\tau, \nabla u)\right)+\tau & =2 \eta \omega D(u),
\end{aligned}\right.
$$

with the coupling parameter $\omega:=1-\frac{\mu}{\lambda} \in(0,1)$. Using dimensionless variables, Oldroyd-B model can be thus described by the system (1.1). For more details of the modeling, c.f., [31, 12] for example.

The theory of Oldroyd-B fluids recently gained quite some attention. According to the coupling strength between the velocity $u$ and the symmetric tensor of the constrains $\tau$, the results on Oldroyd-B model fall into two categories.

(I) Weak coupling case: small coupling parameter $\omega$. The study of the incompressible OldroydB model was started by a pioneering paper given by Guillopé and Saut [15]. They proved that (i) system (1.1) admits a unique local strong solution in suitable Sobolev spaces $H^{s}(\Omega)$ for bounded domains $\Omega \subset \mathbb{R}^{3}$; and (ii) this solution is global provided the data as well as the coupling parameter $\omega$ are sufficiently small. For extensions to this results to the $L^{p}$-setting, see the work of FernandézCara, Guillén and Ortega [13]. The situation of exterior domains was considered first by Hieber, Naito and Shibata [16], where the existence of a unique global strong solution defined in certain function spaces was proved provided the initial data and the coupling parameter $\omega$ are small enough. Another remarkable way to study the Oldroyd-B model (1.1) is constructing solutions in scaling invariant spaces. This approach goes back to the seminal work by Fujita and Kato [14] for the classical incompressible Navier-Stokes equations. While for the Oldroyd-B model, strictly speaking, the system (1.1) does not have any scaling invariance. Fortunately, if we neglect the coupling term $\operatorname{div} \tau$ and the damping term $\tau$, it is found that (1.1) is invariant under the transformation

$$
\left\{\begin{array}{l}
\left(u_{0}, \tau_{0}\right) \rightarrow\left(\ell u_{0}(\ell x), \tau_{0}(\ell x)\right), \\
(u(t, x), \tau(t, x), \Pi(t, x)) \rightarrow\left(\ell u\left(\ell x, \ell^{2} t\right), \tau\left(\ell x, \ell^{2} t\right), \ell^{2} \Pi\left(\ell x, \ell^{2} t\right)\right),
\end{array}\right.
$$

for any $\ell>0$. Chemin and Masmodi [7] first study the Oldroyd-B model (1.1) alone this line. For small coupling parameter $\omega$, they showed the global well-posedness of system (1.1) with initial data $\left(u_{0}, \tau_{0}\right)$ belonging to the critical space $\left(\dot{B}_{p, 1}^{\frac{d}{p}-1}\right)^{d} \times\left(\dot{B}_{p, 1}^{\frac{d}{p}}\right)^{d \times d}$ for any $p \in[1, \infty)$.

(II) Strong coupling case: arbitrary coupling parameter $\omega \in(0,1)$. Oldroyd-B model with strong coupling parameter describes the behavior of the viscoelastic fluids more appropriately. The first result on the system (1.1) with strong coupling parameter was obtained by Molinet and Talhouk [27] for bounded domains. They removed the smallness restriction on the coupling parameter $\omega$ in [15]. For the situation of exterior domains, Hieber and the authors recently [11] improved the main result given in [16] to the situation of non-small coupling parameter. Constructing solutions in scaling invariant spaces with strong coupling is more difficult. Some efforts were made by Chen and Miao in [8], where they established global solutions to the incompressible Oldroyd-B model with small initial data in $B_{2, \infty}^{s}, s>\frac{d}{2}$. Later on, this result was generalized by the second author [34] with data in $\dot{H}^{s} \cap \dot{B}_{2,1}^{\frac{d}{2}},-\frac{d}{2}<s<\frac{d}{2}-1$. It was not until very recently that Zhang and the authors [33] obtained the scaling invariant solution with strong coupling in the critical $L^{p}$ framework. For the weak solutions of incompressible Oldroyd-B fluids, see the work of Lions and Masmoudi [26] for the case $\alpha=0$. The general case $\alpha \neq 0$ is still open up to now. As for the blow-up criterions of the incompressible Oldroyd-B model, there are works [7, 18, 22]. Besides, we would like to mention that Constantin and Kliegl [9] proved the global regularity of solutions in two dimensional case for the Oldroyd-B fluids with diffusive stress. An approach based on Lagrangian particle dynamics can be found in [19, 20, 21, 23, 24, 25, 30, 32]. 
On the other hand, for the classical Navier-Stokes equations, taking advantage of the the algebraical structure of the incompressible condition $\operatorname{div} u=0$, some recent progresses have been made such as [36, 29, 5, 6]. Following these ideas, Zhang [37] proved the existence and uniqueness of solutions to system related to the incompressible viscoelastic fluids with a class of large initial data.

Motivated by [36, 37], the aim of this paper is to investigate the global well-posedness of system (1.1) in critical Besov spaces with the vertical component of the initial velocity field being large. Basically it is the algebraical structure of system (1.1) that enables us to consider this type of initial data. More precisely, using the divergence free condition $\operatorname{div} u=0$, the vertical component of the convection term $u \cdot \nabla u^{d}$ can be rewritten as $u^{h} \partial_{h} u^{d}-u^{d} \operatorname{div}_{h} u^{h}$. As a result, the vertical component $u^{d}$ of the velocity satisfies a linear equation with coefficients depending on the horizontal components $u^{h}$ and $\tau$. This is the reason why we do not believe we have to impose the smallness condition on the initial vertical velocity $u_{0}^{d}$. While the equation for the horizontal velocity $u^{h}$ contains bilinear terms in the horizontal components and also terms describing the interactions between the horizontal components and the vertical one. This necessitates the smallness restriction on the initial horizontal velocity $u_{0}^{h}$. Besides, since the equation of the symmetric tensor of constrains $\tau$ is nonlinear, considering the interactions between the velocity $u$ (with large vertical component) and $\tau$, imposing a smallness condition on $\tau_{0}$ is necessary. In addition, we would like to emphasize that both the equation of vertical velocity and horizontal velocity are linearly coupled with $\tau$, and conversely, the equation of the symmetric tensor of constrains $\tau$ is linearly coupled with $u$ as well. In particular, the coupling parameter $\omega$ is involved. In order to decouple $u$ and $\tau$ linearly, one way to do is simply to apply the estimates for heat equation and transport equation with damping to the velocity $u$ and the symmetric tensor of constrains $\tau$ respectively, and to substitute the estimate for $\tau$ to that for $u$. Nevertheless, in doing this, the coupling parameter $\omega$ must be small enough to close the estimate of $u$. This less interesting weak coupling case is exactly what we want to avoid considering.

Although the strong coupling case with initial data in $L^{p}$ type critical Besov space was investigated by us in [33], large initial vertical velocity is not allowed in [33]. A natural question is that can we solve system (1.1) with strong coupling parameter $\omega$ and large initial vertical velocity $u_{0}^{d}$ ? Before answering it, let us give some heuristic observations. Given large initial vertical velocity, it has been pointed out above that the horizontal velocity $u^{h}$ and the symmetric tensor of constrains $\tau$ should be small. In the circumstances, the linear term $2 \omega D(u)$ should also be small otherwise the equation $(1.1)_{2}$ of $\tau$ can not hold. Since we can not expect the vertical velocity to be small, it is reasonable to construction solutions to system (1.1) with the product $\omega u^{d}$ of the coupling parameter $\omega$ and the vertical velocity $u^{d}$ small. That's why we need the initial product $\omega u_{0}^{d}$ to be small in this paper.

Let us now explain the main ingredients of the proof. Noting that the algebraical structure of system (1.1) plays a key role in our result, the method used in [33] which relied heavily on the Green matrix of the linearized system of (1.1) and was carried our by Lagrangian approach fails to work here. It is because the divergence free condition does not hold anymore in Lagrangian coordinate. In this paper, we exploit energy approach in the critical $L^{2}$ framework. As the damping $\tau$ in the equation $(1.1)_{2}$ may mislead us to the weak coupling case, so a crucial step would be to find a structural mechanism which indicates a parabolic smoothing effect on $u$ in the absence of the damping effect on $\tau$. To the best of our knowledge, in $L^{2}$ framework, researchers [27, 11, 8, 34] were inclined to use the cancelation relation

$$
(\operatorname{div} \tau \mid u)+(D(u) \mid \tau)=0
$$

to eliminate the effect of the coupling parameter $\omega$. The price they had to pay was that the solution space served for $u$ and $\tau$ would be of the same regularity, which violated the scaling. So our strategy is not to estimate $(u, \tau)$ as a whole with an eye to (1.6), but to find a new quantity which obeys the scaling and can be used as a substitute for $\tau$. An ideal candidate is $\mathbb{P} \operatorname{div} \tau$ because on the one hand, $\mathbb{P} \operatorname{div} \tau$ is the exact quantity linearly coupled with $u$ and obeying the scaling. On the other hand, it has 
been shown in [33] that the Green matrix $G(t, x)$ of the following auxiliary system of $(u, \mathbb{P} \operatorname{div} \tau)$

$$
\left\{\begin{aligned}
\operatorname{Re} u_{t}-(1-\omega) \Delta u-\mathbb{P} \operatorname{div} \tau & =-\operatorname{Re} \mathbb{P}(u \cdot \nabla) u, \\
\operatorname{We} \mathbb{P} \operatorname{div} \tau_{t}+\mathbb{P} \operatorname{div} \tau-\omega \Delta u & =-\operatorname{We}\left(\mathbb{P} \operatorname{div}(u \cdot \nabla) \tau+\mathbb{P} \operatorname{div} g_{\alpha}(\tau, \nabla u)\right), \\
u(0) & =u_{0}, \\
\mathbb{P} \operatorname{div} \tau(0) & =\mathbb{P} \operatorname{div} \tau_{0} .
\end{aligned}\right.
$$

behaves just like the heat kernel in the low frequency case. In this paper, we will pay much attention to the system (1.7) in the spirit of Danchin [10]. More precisely, we first localize (1.7) in frequencies according to the Littlewood-Paley decomposition. Then a variant energy method will be used to estimate each dyadic block $\left(\dot{\Delta}_{q} u, \dot{\Delta}_{q} \mathbb{P} \operatorname{div} \tau\right)$. Owing to the fact that the linear operator associated with (1.7) can not be diagonalized in a basis independent of $\xi$, coercive estimates can not be achieved by standard energy argument. Since the parabolic-hyperbolic system (1.7) behaves differently in high and low frequencies, we shall consider the following quantity (for some $q_{0}, q_{1} \in \mathbb{Z}$ to be chosen later):

$$
Y_{q}= \begin{cases}{\left[\left\|u_{q}\right\|_{L^{2}}^{2}+2\left\|\frac{\mathrm{We}}{\mathrm{Re}} \mathbb{P} \operatorname{div} \tau_{q}\right\|_{L^{2}}^{2}+2\left(u_{q} \mid \frac{\mathrm{We}}{\mathrm{Re}} \mathbb{P} \operatorname{div} \tau_{q}\right)+2\left(u_{q} \mid \frac{\mathrm{We}}{\operatorname{Re}} \Delta \mathbb{P} \operatorname{div} \tau_{q}\right)\right]^{\frac{1}{2}},} & \text { for } \quad q \leq q_{1}, \\ {\left[2\left\|u_{q}\right\|_{L^{2}}^{2}+\left\|\frac{(1-\omega) \mathrm{We}}{\omega \operatorname{Re}} \mathbb{P} \operatorname{div} \tau_{q}\right\|_{L^{2}}^{2}-2\left(u_{q} \mid \frac{(1-\omega) \mathrm{We}}{\omega \operatorname{Re}} \mathbb{P} \operatorname{div} \tau_{q}\right)\right]^{\frac{1}{2}},} & \text { for } \quad q>q_{0}, \\ {\left[\left\|u_{q}\right\|_{L^{2}}^{2}+\frac{\mathrm{We}}{\omega \operatorname{Re}}\left\|\Lambda^{-1} \mathbb{P} \operatorname{div} \tau_{q}\right\|_{L^{2}}^{2}\right]^{\frac{1}{2}},} & \text { for } \quad q_{1}<q \leq q_{0} .\end{cases}
$$

Some words about the definition of $Y_{q}$ are in order.

- For $q \leq q_{1}$, the parabolic smoothing effect of $\mathbb{P} \operatorname{div} \tau$ is obtained with the aid of the linear coupling term $-\mathbb{P} \operatorname{div} \tau$ in $(1.7]_{1}$, that's why we introduce the cross term $\left(u_{q} \mid \frac{\mathrm{We}}{\operatorname{Re}} \Delta \mathbb{P} \operatorname{div} \tau_{q}\right)$.

- For $q>q_{0}$, compared with the linear coupling term $-\mathbb{P} \operatorname{div} \tau$ in $(1.7)_{1}$, the damping term $\mathbb{P} \operatorname{div} \tau$ in $(1.7)_{2}$ is predominate, and thus there is a damping effect on $\mathbb{P} \operatorname{div} \tau$.

- For $q_{1}<q \leq q_{0}$, a new quantity $\Lambda^{-1} \mathbb{P} \operatorname{div} \tau$ is introduced to fill up the gap between $q_{1}$ and $q_{0}$. Indeed, in this case, we do not need to care about the scaling thanks to Bernstein's inequalities. On this basis, the cancelation relation (1.6) motivates us to choose $\Lambda^{-1} \mathbb{P} \operatorname{div} \tau$ to replace $\mathbb{P} \operatorname{div} \tau$. In doing so, $u$ and $\Lambda^{-1} \mathbb{P} \operatorname{div} \tau$ are linearly decoupled, and $\Lambda^{-1} \mathbb{P} \operatorname{div} \tau$ also possesses a damping effect because of the damping term. Therefore, in this paper we combine the case $q>q_{0}$ with $q_{1}<q \leq q_{0}$, and regard them as high frequency. The left part $q \leq q_{1}$ is regarded as low frequency.

We would like to remark that similar construction of $Y_{q}$ was also used by the second author in [35] for the compressible Oldroyd-B model. However, for $q_{1}<q \leq q_{0}$, the quantity in [35] corresponding to $Y_{q}$ is

$$
\tilde{Y}_{q}:=\left[\left\|\mathbb{P} u_{q}\right\|_{L^{2}}^{2}+\frac{\mathrm{We}}{\omega \operatorname{Re}}\left\|\Lambda^{-1} \mathbb{P} \operatorname{div} \tau_{q}\right\|_{L^{2}}^{2}-\frac{2 \beta(1-\omega) \mathrm{We}}{\omega \operatorname{Re}}\left(\mathbb{P} u_{q} \mid \mathbb{P} \operatorname{div} \tau_{q}\right)\right]^{\frac{1}{2}},
$$

with some positive constant $\beta$. Since the cross term $\left(\mathbb{P} u_{q} \mathbb{P} \operatorname{div} \tau_{q}\right)$ is involved in $\tilde{Y}_{q}, \Lambda^{-1} \mathbb{P} \operatorname{div} \tau$ in [35] exhibits the parabolic smoothing effect stemming from the linear coupling term $-\mathbb{P} \operatorname{div} \tau$. Hence the case $q_{1}<q \leq q_{0}$ is regarded as low frequency in [35]. The point is, though, that $\tilde{Y}_{q}$ can not be used in this paper to replace $Y_{q}$ because the parabolic smoothing effect for $\Lambda^{-1} \mathbb{P} \operatorname{div} \tau$ and hence for $u$ is too weak (depending on $\omega$ ) to deal with the linear coupling term $2 \omega D(u)$ in $\left.{ }_{1.1}\right)_{2}$.

Obviously, the above analysis do not take into account the anisotropy. In order to distinguish the velocity $u$ from different directions, we must bound $\left(u^{h},(\mathbb{P} \operatorname{div} \tau)^{h}\right)$ and $\left(u^{d},(\mathbb{P} \operatorname{div} \tau)^{d}\right)$ separately (please refer to Section 3 for more details).

We shall obtain the existence and uniqueness of a solution $(u, \tau)$ to 1.1 in the following space.

Definition 1.1. For $T>0$, and $s \in \mathbb{R}$, let us denote

$$
\mathcal{E}_{T}^{s}:=\left(\tilde{C}_{T}\left(\dot{B}_{2,1}^{s-1}\right) \cap L_{T}^{1}\left(\dot{B}_{2,1}^{s+1}\right)\right)^{d} \times\left(\tilde{C}_{T}\left(\dot{B}_{2,1}^{s}\right) \cap L_{T}^{1}\left(\dot{B}_{2,1}^{s}\right)\right)^{d \times d} .
$$


We use the notation $\mathcal{E}^{s}$ if $T=\infty$, changing $[0, T]$ into $[0, \infty)$ in the definition above.

Our main result is stated as follows:

Theorem 1.1. Let $d \geq 2$. Assume that $\left(u_{0}, \tau_{0}\right) \in\left(\dot{B}_{2,1}^{\frac{d}{2}-1}\right)^{d} \times\left(\dot{B}_{2,1}^{\frac{d}{2}}\right)^{d \times d}$ with $\operatorname{div} u_{0}=0$. There exists a positive constant $M$, depending on $d, \operatorname{Re}$ and $\mathrm{We}$, but independent of $\omega$,such that if

$$
\frac{C_{0}}{(1-\omega)^{9}}\left(\left\|u_{0}^{h}\right\|_{\dot{B}_{2,1}^{\frac{d}{2}-1}}+\omega\left\|u_{0}^{d}\right\|_{\dot{B}_{2,1}^{\frac{d}{2}-1}}+\left\|\tau_{0}\right\|_{\dot{B}_{2,1}^{\frac{d}{2}-1}}\right) \exp \left(\frac{C_{0}}{(1-\omega)^{12}}\left\|u_{0}^{d}\right\|_{\dot{B}_{2,1}^{\frac{d}{2}-1}}^{2}\right) \leq 1,
$$

then system (1.1) admits a unique global solution $(u, \tau)$ in $\mathcal{E}^{\frac{d}{2}}$ with

$$
\begin{aligned}
& \left\|u^{d}\right\|_{\tilde{L}_{t}^{\infty}\left(\dot{B}_{2,1}^{\frac{d}{2}-1}\right)}+\left\|(\mathbb{P} \operatorname{div} \tau)^{d}\right\|_{\tilde{L}_{t}^{\infty}\left(\dot{B}_{2,1}^{\frac{d}{2}-1}\right)}+\left\|u^{d}\right\|_{L_{t}^{1}\left(\dot{B}_{2,1}^{\frac{d}{2}+1}\right)}+\left\|(\mathbb{P} \operatorname{div} \tau)^{d}\right\|_{L_{t}^{1}\left(\dot{B}_{2,1}^{\frac{d}{2}+1, \frac{d}{2}-1}\right)} \\
& \leq \frac{C_{0}}{(1-\omega)^{3}}\left(1+\left\|u_{0}^{d}\right\|_{\dot{B}_{2,1}^{\frac{d}{2}-1}}+\left\|(\mathbb{P} \operatorname{div} \tau)_{0}^{d}\right\|_{\dot{B}_{2,1}^{\frac{d}{2}-1}}\right),
\end{aligned}
$$

and

$$
\begin{aligned}
& \left\|u^{h}\right\|_{\tilde{L}_{t}^{\infty}\left(\dot{B}_{2,1}^{\left.\frac{d}{2}-1\right)}\right.}+\left\|(\mathbb{P} \operatorname{div} \tau)^{h}\right\|_{\left.\tilde{L}_{t}^{\infty} \dot{B}_{2,1}^{\frac{d}{2}-1}\right)}+\left\|u^{h}\right\|_{L_{t}^{1}\left(\dot{B}_{2,1}^{\frac{d}{2}+1}\right)}+\left\|(\mathbb{P} \operatorname{div} \tau)^{h}\right\|_{L_{t}^{1}\left(\dot{B}_{2,1}^{\frac{d}{2}+1, \frac{d}{2}-1}\right)} \\
\leq & \frac{C_{0}}{(1-\omega)^{6}}\left(\left\|u_{0}^{h}\right\|_{\dot{B}_{2,1}^{\frac{d}{2}-1}}+\omega\left\|u_{0}^{d}\right\|_{\dot{B}_{2,1}^{\frac{d}{2}-1}}+\left\|\tau_{0}\right\|_{\dot{B}_{2,1}^{\frac{d}{2}-1}}\right) \exp \left(\frac{C_{0}}{(1-\omega)^{12}}\left\|u_{0}^{d}\right\|_{\dot{B}_{2,1}^{2}}^{2}\right)
\end{aligned}
$$

and

$$
\begin{aligned}
& \|\tau\|_{\tilde{L}_{t}^{\infty}\left(\dot{B}_{2,1}^{2}\right)}+\|\tau\|_{L_{t}^{1}\left(\dot{B}_{2,1}^{\frac{d}{2}}\right)} \\
\leq & \frac{C_{0}}{(1-\omega)^{6}}\left(\left\|u_{0}^{h}\right\|_{\dot{B}_{2,1}^{\frac{d}{2}-1}}+\omega\left\|u_{0}^{d}\right\|_{\dot{B}_{2,1}^{\frac{d}{2}-1}}+\left\|\tau_{0}\right\|_{\dot{B}_{2,1}^{\frac{d}{2}-1}}\right) \exp \left(\frac{C_{0}}{(1-\omega)^{12}}\left\|u_{0}^{d}\right\|_{\dot{B}_{2,1}^{\frac{d}{2}-1}}^{2}\right) .
\end{aligned}
$$

The rest part of this paper is organized as follows. In Section 2, we introduce the tools ( the Littlewood-Paley decomposition and paradifferertial calculus) and give some nonlinear estimates in Besov spaces. Section 3 is devoted to the global a priori estimates of system (1.1). The proof of Theorem 1.1 is given in Section 4.

\section{Notation.}

(1) For $f \in \mathcal{S}^{\prime}, f_{q}:=\dot{\Delta}_{q} f$.

(2) For $x=\left(x_{1}, \cdots, x_{d}\right)$, we denote $x_{h}:=\left(x_{1}, \cdots, x_{d-1}\right)$; For vector field $u(x)=\left(u^{1}(x), \cdots, u^{d}(x)\right)$, we denote $u^{h}(x):=\left(u^{1}(x), \cdots, u^{d-1}(x)\right), \partial_{h} u(x):=\left(\partial_{1} u(x), \cdots, \partial_{d-1} u(x)\right)^{\top}$, and $\operatorname{div}_{h} u^{h}(x):=$ $\partial_{1} u^{1}(x)+\cdots+\partial_{d-1} u^{d-1}(x)$.

(3) For $a, b \in L^{2},(a \mid b)$ denotes the $L^{2}$ inner product of $a$ and $b$.

(4) Throughout the paper, $C$ denotes various "harmless" positive constants, and we sometimes use the notation $A \lesssim B$ as an equivalent to $A \leq C B$. The notation $A \approx B$ means that $A \lesssim B$ and $B \lesssim A$.

\section{The Functional Tool Box}

The results of the present paper rely on the use of a dyadic partition of unity with respect to the Fourier variables, the so-called the Littlewood-Paley decomposition. Let us briefly explain how it may be built in the case $x \in \mathbb{R}^{d}$ which the readers may see more details in [1, 3]. Let $(\chi, \varphi)$ be a couple of $C^{\infty}$ functions satisfying

$$
\operatorname{Supp} \chi \subset\left\{|\xi| \leq \frac{4}{3}\right\}, \quad \operatorname{Supp} \varphi \subset\left\{\frac{3}{4} \leq|\xi| \leq \frac{8}{3}\right\}
$$


and

$$
\begin{gathered}
\chi(\xi)+\sum_{q \geq 0} \varphi\left(2^{-q} \xi\right)=1, \\
\sum_{q \in \mathbb{Z}} \varphi\left(2^{-q} \xi\right)=1, \quad \text { for } \quad \xi \neq 0 .
\end{gathered}
$$

Set $\varphi_{q}(\xi)=\varphi\left(2^{-q} \xi\right), h_{q}=\mathcal{F}^{-1}\left(\varphi_{q}\right)$, and $\tilde{h}=\mathcal{F}^{-1}(\chi)$. The dyadic blocks and the low-frequency cutoff operators are defined for all $q \in \mathbb{Z}$ by

Then

$$
\begin{aligned}
& \dot{\Delta}_{q} u=\varphi\left(2^{-q} \mathrm{D}\right) u=\int_{\mathbb{R}^{d}} h_{q}(y) u(x-y) d y, \\
& \dot{S}_{q} u=\chi\left(2^{-q} \mathrm{D}\right) u=\int_{\mathbb{R}^{d}} \tilde{h}_{q}(y) u(x-y) d y .
\end{aligned}
$$

$$
u=\sum_{q \in \mathbb{Z}} \Delta_{q} u
$$

holds for tempered distributions modulo polynomials. As working modulo polynomials is not appropriate for nonlinear problems, we shall restrict our attention to the set $\mathcal{S}_{h}^{\prime}$ of tempered distributions $u$ such that

$$
\lim _{q \rightarrow-\infty}\left\|\dot{S}_{q} u\right\|_{L^{\infty}}=0
$$

Note that (2.2) holds true whenever $u$ is in $\mathcal{S}_{h}^{\prime}$ and that one may write

$$
\dot{S}_{q} u=\sum_{p \leq q-1} \dot{\Delta}_{p} u
$$

Besides, we would like to mention that the Littlewood-Paley decomposition has a nice property of quasi-orthogonality:

$$
\dot{\Delta}_{p} \dot{\Delta}_{q} u \equiv 0 \text { if }|p-q| \geq 2 \text { and } \dot{\Delta}_{p}\left(\dot{S}_{q-1} u \dot{\Delta}_{q} u\right) \equiv 0 \text { if }|p-q| \geq 5 .
$$

One can now give the definition of homogeneous Besov spaces.

Definition 2.1. For $s \in \mathbb{R},(p, r) \in[1, \infty]^{2}$, and $u \in \mathcal{S}^{\prime}\left(\mathbb{R}^{d}\right)$, we set

$$
\|u\|_{\dot{B}_{p, r}^{s}}=\left\|2^{s q}\right\| \dot{\Delta}_{q} u\left\|_{L^{p}}\right\|_{\ell^{r}}
$$

We then define the space $\dot{B}_{p, r}^{s}:=\left\{u \in \mathcal{S}_{h}^{\prime}\left(\mathbb{R}^{d}\right),\|u\|_{\dot{B}_{p, r}^{s}}<\infty\right\}$.

Since homogeneous Besov spaces fail to have nice inclusion properties, it is wise to define hybrid Besov spaces where the growth conditions satisfied by the dyadic blocks are different for low and high frequencies. In fact, hybrid Besov spaces played a crucial role for proving global well-posedness of compressible barotropic Navier-Stokes equations in critical spaces [10]. Let us now define the hybrid Besov spaces that we need. Here our notations are somehow different from those in [10].

Definition 2.2. Let $s, t \in \mathbb{R}$, and $u \in \mathcal{S}^{\prime}\left(\mathbb{R}^{d}\right)$. For some fixed $q_{0} \in \mathbb{Z}$, we set

$$
\|u\|_{\dot{B}_{2,1}^{s, t}}:=\sum_{q \leq q_{0}} 2^{q s}\left\|\dot{\Delta}_{q} u\right\|_{L^{2}}+\sum_{q>q_{0}} 2^{q t}\left\|\dot{\Delta}_{q} u\right\|_{L^{2}} .
$$

We then define the space $\dot{B}_{2,1}^{s, t}:=\left\{u \in \mathcal{S}_{h}^{\prime}\left(\mathbb{R}^{d}\right),\|u\|_{\dot{B}_{2,1}^{s, t}}<\infty\right\}$.

Remark 2.1. For all $s, t \in \mathbb{R}, \tilde{q}_{0} \in \mathbb{Z}$, and $u \in \mathcal{S}^{\prime}\left(\mathbb{R}^{d}\right)$, setting

$$
\|u\|_{\tilde{B}_{2,1}^{s, t}}:=\sum_{q \leq \tilde{q}_{0}} 2^{q s}\left\|\dot{\Delta}_{q} u\right\|_{L^{2}}+\sum_{q>\tilde{q}_{0}} 2^{q t}\left\|\dot{\Delta}_{q} u\right\|_{L^{2}},
$$

then it is easy to verify that $\|u\|_{\tilde{B}_{2,1}^{s, t}} \approx\|u\|_{\dot{B}_{2,1}^{s, t}}$.

The following lemma describes the way derivatives act on spectrally localized functions. 
Lemma 2.1 (Bernstein's inequalities). Let $k \in \mathbb{N}$ and $0<r<R$. There exists a constant $C$ depending on $r, R$ and $d$ such that for all $(a, b) \in[1, \infty]^{2}$, we have for all $\lambda>0$ and multi-index $\alpha$

- If $\operatorname{Supp} \hat{f} \subset B(0, \lambda R)$, then $\sup _{\alpha=k}\left\|\partial^{\alpha} f\right\|_{L^{b}} \leq C^{k+1} \lambda^{k+d\left(\frac{1}{a}-\frac{1}{b}\right)}\|f\|_{L^{a}}$.

- If $\operatorname{Supp} \hat{f} \subset C(0, \lambda r, \lambda R)$, then $C^{-k-1} \lambda^{k}\|f\|_{L^{a}} \leq \sup _{|\alpha|=k}\left\|\partial^{\alpha} f\right\|_{L^{a}} \leq C^{k+1} \lambda^{k}\|f\|_{L^{a}}$

In fact, the following special case of Lemma 2.1 will be used very frequently in this paper.

Corollary 2.1. Let $\Lambda:=\sqrt{-\Delta}$, then for any $f \in \mathcal{S}^{\prime}$, there holds

$$
\frac{3}{4} 2^{q}\left\|\dot{\Delta}_{q} f\right\|_{L^{2}} \leq\left\|\Lambda \dot{\Delta}_{q} f\right\|_{L^{2}} \leq \frac{8}{3} 2^{q}\left\|\dot{\Delta}_{q} f\right\|_{L^{2}} .
$$

Owing to the property (2.1) of $\operatorname{Supp} \varphi$, (2.4) can be easily obtained by virtue of Plancherel's Theorem.

Let us now state some classical properties for the Besov spaces.

Proposition 2.1. For all $s, s_{1}, s_{2} \in \mathbb{R}, 1 \leq p, p_{1}, p_{2}, r, r_{1}, r_{2} \leq \infty$, the following properties hold true:

- If $p_{1} \leq p_{2}$ and $r_{1} \leq r_{2}$, then $\dot{B}_{p_{1}, r_{1}}^{s} \hookrightarrow \dot{B}_{p_{2}, r_{2}}^{s-\frac{d}{p_{1}}+\frac{d}{p_{2}}}$.

- If $s_{1} \neq s_{2}$ and $\theta \in(0,1),\left[\dot{B}_{p, r_{1}}^{s_{1}}, \dot{B}_{p, r_{2}}^{s_{2}}\right]_{(\theta, r)}=\dot{B}_{p, r}^{\theta s_{1}+(1-\theta) s_{2}}$.

- For any smooth homogeneous of degree $m \in \mathbb{Z}$ function $F$ on $\mathbb{R}^{d} \backslash\{0\}$, the operator $F(D)$ maps $\dot{B}_{p, r}^{s}$ in $\dot{B}_{p, r}^{s-m}$.

Next we recall a few nonlinear estimates in Besov spaces which may be obtained by means of paradifferential calculus. Firstly introduced by J. M. Bony in [2], the paraproduct between $f$ and $g$ is defined by

$$
\dot{T}_{f} g=\sum_{q \in \mathbb{Z}} \dot{S}_{q-1} f \dot{\Delta}_{q} g
$$

and the remainder is given by

$$
\dot{R}(f, g)=\sum_{q \geq-1} \dot{\Delta}_{q} f \tilde{\dot{\Delta}}_{q} g
$$

with

$$
\tilde{\dot{\Delta}}_{q} g=\left(\dot{\Delta}_{q-1}+\dot{\Delta}_{q}+\dot{\Delta}_{q+1}\right) g .
$$

We have the following so-called Bony's decomposition:

$$
f g=\dot{T}_{f} g+\dot{T}_{g} f+\dot{R}(f, g)=\dot{T}_{f} g+\dot{T}_{g}^{\prime} f
$$

where $\dot{T}_{g}^{\prime} f:=\dot{T}_{g} f+\dot{R}(f, g)$. The paraproduct $\dot{T}$ and the remainder $\dot{R}$ operators satisfy the following continuous properties.

Proposition 2.2. For all $s \in \mathbb{R}, \sigma>0$, and $1 \leq p, p_{1}, p_{2}, r, r_{1}, r_{2} \leq \infty$, the paraproduct $\dot{T}$ is a bilinear, continuous operator from $L^{\infty} \times \dot{B}_{p, r}^{s}$ to $\dot{B}_{p, r}^{s}$ and from $\dot{B}_{\infty, r_{1}}^{-\sigma} \times \dot{B}_{p, r_{2}}^{s}$ to $\dot{B}_{p, r}^{s-\sigma}$ with $\frac{1}{r}=\min \left\{1, \frac{1}{r_{1}}+\frac{1}{r_{2}}\right\}$. The remainder $\dot{R}$ is bilinear continuous from $\dot{B}_{p_{1}, r_{1}}^{s_{1}} \times \dot{B}_{p_{2}, r_{2}}^{s_{2}}$ to $\dot{B}_{p, r}^{s_{1}+s_{2}}$ with $s_{1}+s_{2}>0, \frac{1}{p}=\frac{1}{p_{1}}+\frac{1}{p_{2}} \leq 1$, and $\frac{1}{r}=\frac{1}{r_{1}}+\frac{1}{r_{2}} \leq 1$.

In view of (2.5), Proposition 2.2 and Bernstein's inequalities, one easily deduces the following product estimates:

Corollary 2.2. Let $p \in[1, \infty]$. If $s_{1}, s_{2} \leq \frac{d}{p}$ and $s_{1}+s_{2}>d \max \left\{0, \frac{2}{p}-1\right\}$, then there holds

$$
\|u v\|_{\dot{B}_{p, 1}^{s_{1}+s_{2}-\frac{d}{p}}} \leq C\|u\|_{\dot{B}_{p, 1}^{s_{1}}}\|v\|_{\dot{B}_{p, 1}^{s_{2}}} .
$$

In the following, we shall give a commutator estimate, which will be used to deal with the convection terms.

Lemma $2.2([35])$. Let $\Lambda:=\sqrt{-\Delta}$, then

$$
\left\|\left[\Lambda^{-1}, \dot{S}_{q-1} v \cdot \nabla\right] \dot{\Delta}_{q} u\right\|_{L^{2}} \leq C\left\|\nabla \dot{S}_{q-1} v\right\|_{L^{\infty}}\left\|\Lambda^{-1} \dot{\Delta}_{q} u\right\|_{L^{2}} .
$$


The divergence free condition allows us to get the following lemma, which plays a key role in this paper.

Lemma 2.3 ([37]). Let $v$ be a divergence free vector field. We then have

$$
\left\|v^{d}\right\|_{L_{x_{h}}^{2}\left(L_{x_{d}}^{\infty}\right)}^{2} \leq C\left\|\operatorname{div}_{h} v^{h}\right\|_{L^{2}}\left\|v^{d}\right\|_{L^{2}}, \quad \text { and } \quad\left\|v^{d}\right\|_{L^{\infty}} \leq C\left\|v^{h}\right\|_{\dot{B}_{2,1}^{\frac{d}{2}}}^{\frac{1}{2}}\left\|v^{d}\right\|_{\dot{B}_{2,1}^{\frac{d}{2}}}^{\frac{1}{2}} \text {. }
$$

The study of non-stationary PDEs requires spaces of the type $L_{T}^{\rho}(X)=L^{\rho}(0, T ; X)$ for appropriate Banach spaces $X$. In our case, we expect $X$ to be a Besov space, so that it is natural to localize the equations through Littlewood-Paley decomposition. We then get estimates for each dyadic block and perform integration in time. But, in doing so, we obtain the bounds in spaces which are not of the type $L^{\rho}\left(0, T ; \dot{B}_{p, r}^{s}\right)$. That naturally leads to the following definition introduced by Chemin and Lerner in [4].

Definition 2.3. For $\rho \in[1,+\infty], s \in \mathbb{R}$, and $T \in(0,+\infty)$, we set

$$
\|u\|_{\tilde{L}_{T}^{\rho}\left(\dot{B}_{p, r}^{s}\right)}=\left\|2^{q s}\right\| \dot{\Delta}_{q} u(t)\left\|_{L_{T}^{\rho}\left(L^{p}\right)}\right\|_{\ell^{r}}
$$

and denote by $\tilde{L}_{T}^{\rho}\left(\dot{B}_{p, r}^{s}\right)$ the subset of distributions $u \in \mathcal{S}^{\prime}\left([0, T] \times \mathbb{R}^{N}\right)$ with finite $\|u\|_{\tilde{L}_{T}^{\rho}\left(\dot{B}_{p, r}^{s}\right)}$ norm. When $T=+\infty$, the index $T$ is omitted. We further denote $\tilde{C}_{T}\left(\dot{B}_{p, r}^{s}\right)=C\left([0, T] ; \dot{B}_{p, r}^{s}\right) \cap \tilde{L}_{T}^{\infty}\left(\dot{B}_{p, r}^{s}\right)$.

Remark 2.2. All the properties of continuity for the paraproduct, remainder, and product remain true for the Chemin-Lerner spaces. The exponent $\rho$ just has to behave according to Hölder's ineauality for the time variable.

Remark 2.3. The spaces $\tilde{L}_{T}^{\rho}\left(\dot{B}_{p, r}^{s}\right)$ can be linked with the classical space $L_{T}^{\rho}\left(\dot{B}_{p, r}^{s}\right)$ via the Minkowski inequality:

$$
\|u\|_{\tilde{L}_{T}^{\rho}\left(\dot{B}_{p, r}^{s}\right)} \leq\|u\|_{L_{T}^{\rho}\left(\dot{B}_{p, r}^{s}\right)} \quad \text { if } \quad r \geq \rho, \quad\|u\|_{\tilde{L}_{T}^{\rho}\left(\dot{B}_{p, r}^{s}\right)} \geq\|u\|_{L_{T}^{\rho}\left(\dot{B}_{p, r}^{s}\right)} \quad \text { if } \quad r \leq \rho .
$$

\section{A Priori Estimates}

We begin this section by rewriting the system (1.1) in term of $\left(u^{i},(\mathbb{P} \operatorname{div} \tau)^{i}\right), 1 \leq i \leq d$, as follows

$$
\left\{\begin{array}{l}
\partial_{t} u^{i}-\frac{1-\omega}{\operatorname{Re}} \Delta u^{i}-\frac{1}{\operatorname{Re}}(\mathbb{P} \operatorname{div} \tau)^{i}=f^{i}, \\
\partial_{t}(\mathbb{P} \operatorname{div} \tau)^{i}+\frac{1}{\operatorname{We}}(\mathbb{P} \operatorname{div} \tau)^{i}-\frac{\omega}{\mathrm{We}} \Delta u^{i}=h^{i},
\end{array}\right.
$$

where $f^{i}:=-(\mathbb{P}(u \cdot \nabla) u)^{i}$, and $h^{i}:=-\left(\mathbb{P} \operatorname{div}(u \cdot \nabla) \tau+\mathbb{P} \operatorname{div} g_{\alpha}(\tau, \nabla u)\right)^{i}$. For simplicity, let us denote $\sigma:=\mathbb{P} \operatorname{div} \tau$, then system (3.1) can be localized as

$$
\left\{\begin{array}{l}
\partial_{t} u_{q}^{i}+\dot{S}_{q-1} u \cdot \nabla u_{q}^{i}-\frac{1-\omega}{\operatorname{Re}} \Delta u_{q}^{i}-\frac{1}{\operatorname{Re}} \sigma_{q}^{i}=\tilde{f}_{q}^{i}, \\
\partial_{t} \sigma_{q}^{i}+\dot{S}_{q-1} u \cdot \nabla \sigma_{q}^{i}+\frac{1}{\mathrm{We}} \sigma_{q}^{i}-\frac{\omega}{\mathrm{We}} \Delta u_{q}^{i}=\tilde{h}_{q}^{i},
\end{array}\right.
$$

where $\tilde{f}_{q}^{i}:=\dot{S}_{q-1} u \cdot \nabla u_{q}^{i}+f_{q}^{i}$, and $\tilde{h}_{q}^{i}:=\dot{S}_{q-1} u \cdot \nabla \sigma_{q}^{i}+h_{q}^{i}$.

Denote

$$
\begin{aligned}
& A^{h}(t):=\left\|u^{h}\right\|_{\tilde{L}_{t}^{\infty}\left(\dot{B}_{2,1}^{\left.\frac{d}{2}-1\right)}\right.}+\left\|\sigma^{h}\right\|_{\tilde{L}_{t}^{\infty}\left(\dot{B}_{2,1}^{\frac{d}{2}-1}\right)}+\left\|u^{h}\right\|_{L_{t}^{1}\left(\dot{B}_{2,1}^{\frac{d}{2}+1}\right)}+\left\|\sigma^{h}\right\|_{L_{t}^{1}\left(\dot{B}_{2,1}^{\frac{d}{2}+1, \frac{d}{2}-1}\right)}, \\
& A^{d}(t):=\left\|u^{d}\right\|_{\tilde{L}_{t}^{\infty}\left(\dot{B}_{2,1}^{\frac{d}{2}-1}\right)}+\left\|\sigma^{d}\right\|_{\tilde{L}_{t}^{\infty}\left(\dot{B}_{2,1}^{\frac{d}{2}-1}\right)}+\left\|u^{d}\right\|_{L_{t}^{1}\left(\dot{B}_{2,1}^{\frac{d}{2}+1}\right)}+\left\|\sigma^{d}\right\|_{L_{t}^{1}\left(\dot{B}_{2,1}^{\frac{d}{2}+1, \frac{d}{2}-1}\right)}, \\
& A^{h}(0):=\left\|u_{0}^{h}\right\|_{\dot{B}_{2,1}^{\frac{d}{2}-1}}+\left\|\sigma_{0}^{h}\right\|_{\dot{B}_{2,1}^{\frac{d}{2}-1}}, \quad A^{d}(0):=\left\|u_{0}^{d}\right\|_{\dot{B}_{2,1}^{\frac{d}{2}-1}}+\left\|\sigma_{0}^{d}\right\|_{\dot{B}_{2,1}^{\frac{d}{2}-1}}, \\
& B(t):=\left\|\left.\tau\right|_{\tilde{L}_{t}^{\infty}\left(\dot{B}_{2,1}^{2}\right)}+\right\| \tau\left\|_{L_{t}^{1}\left(\dot{B} \dot{B}_{2,1}^{2}\right)}, \quad B(0):=\right\| \tau_{0} \|_{\dot{B}_{2,1}^{\frac{d}{2}}} .
\end{aligned}
$$

We will establish the following proposition. 
Proposition 3.1. Let $(u, \tau)$ be a solution to system (1.1). There exist constants $C_{1}, C_{2}$ and $C_{3}$ depending on $d, \operatorname{Re}$, and We but independent of $\omega$, such that the following inequalities hold

$$
\begin{aligned}
& A^{h}(t) \leq \frac{C_{1}}{(1-\omega)^{3}}\left(A^{h}(0)+\int_{0}^{t}\left(\left\|u^{h}\right\|_{\dot{B}_{2,1}^{\frac{d}{2}+1}}+\left\|u^{d}\right\|_{\dot{B}_{2,1}^{\frac{d}{2}+1}}\right)\|\tau\|_{\dot{B}_{2,1}^{\frac{d}{2}}} d t^{\prime}\right. \\
& +\int_{0}^{t}\left(\left\|u^{h}\right\|_{\dot{B}_{2,1}^{\frac{d}{2}-1}}\left\|u^{h}\right\|_{\dot{B}_{2,1}^{\frac{d}{2}+1}}+\left\|u^{h}\right\|_{\dot{B}_{2,1}^{\frac{d}{2}+1}}^{\frac{1}{2}}\left\|u^{d}\right\|_{\dot{B}_{2,1}^{\frac{d}{2}-1}}^{\frac{1}{2}}\left\|u^{h}\right\|_{\dot{B}_{2,1}^{\frac{d}{2}-1}}^{\frac{1}{2}}\left\|u^{d}\right\|_{\dot{B}_{2,1}^{\frac{d}{2}+1}}^{\frac{1}{2}}\right) d t^{\prime} \\
& \left.+\int_{0}^{t}\left\|u^{h}\right\|_{\dot{B}_{2,1}^{\frac{d}{2}+1}}^{\frac{1}{2}}\left\|u^{d}\right\|_{\dot{B}_{2,1}^{\frac{d}{2}+1}}^{\frac{1}{2}}\left\|u^{h}\right\|_{\dot{B}_{2,1}^{\frac{d}{2}-1}} d t^{\prime}\right) \\
& A^{d}(t) \leq \frac{C_{2}}{(1-\omega)^{3}}\left(A^{d}(0)+\int_{0}^{t}\left(\left\|u^{h}\right\|_{\dot{B}_{2,1}^{\frac{d}{2}+1}}+\left\|u^{d}\right\|_{\dot{B}_{2,1}^{\frac{d}{2}+1}}\right)\|\tau\|_{\dot{B}_{2,1}^{\frac{d}{2}}} d t^{\prime}\right. \\
& +\int_{0}^{t}\left(\left\|u^{h}\right\|_{\dot{B}_{2,1}^{\frac{d}{2}-1}}\left\|u^{h}\right\|_{\dot{B}_{2,1}^{\frac{d}{2}+1}}+\left\|u^{h}\right\|_{\dot{B}_{2,1}^{\frac{d}{2}+1}}^{\frac{1}{2}}\left\|u_{\dot{B}_{2,1}^{\frac{d}{2}-1}}^{\frac{1}{2}}\right\| u_{\dot{B}_{2,1}^{h}}^{\frac{1}{2}}\left\|u_{\dot{B}_{2,1}^{\frac{d}{2}-1}}^{\frac{1}{2}}\right\|^{\frac{1}{2}}\right) d t^{\prime} \\
& \left.+\int_{0}^{t}\left(\left\|u^{h}\right\|_{\dot{B}_{2,1}^{\frac{d}{2}+1}}+\left\|u^{h}\right\|_{\dot{B}_{2,1}^{\frac{d}{2}+1}}^{\frac{1}{2}}\left\|u^{d}\right\|_{\dot{B}_{2,1}^{\frac{d}{2}+1}}^{\frac{1}{2}}\right)\left\|u^{d}\right\|_{\dot{B}_{2,1}^{\frac{d}{2}-1}} d t^{\prime}\right) \text {, }
\end{aligned}
$$

and

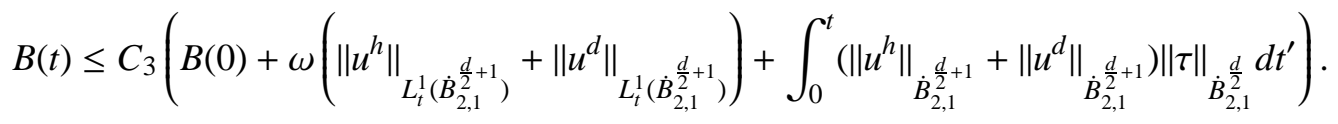

Proof. For $1 \leq i \leq d$, let us denote

$$
\begin{gathered}
Y_{q}^{i}:=\left[\left\|u_{q}^{i}\right\|_{L^{2}}^{2}+2\left\|\frac{\mathrm{We}}{\operatorname{Re}} \sigma_{q}^{i}\right\|_{L^{2}}^{2}+2\left(u_{q}^{i} \mid \frac{\mathrm{We}}{\operatorname{Re}} \sigma_{q}^{i}\right)+2\left(u_{q}^{i} \mid \frac{\mathrm{We}}{\operatorname{Re}} \Delta \sigma_{q}^{i}\right)\right]^{\frac{1}{2}}, \quad \text { for } \quad q \leq q_{1}, \\
Y_{q}^{i}:=\left[2\left\|u_{q}^{i}\right\|_{L^{2}}^{2}+\left\|\frac{(1-\omega) \mathrm{We}}{\omega \operatorname{Re}} \sigma_{q}^{i}\right\|_{L^{2}}^{2}-2\left(u_{q}^{i} \frac{(1-\omega) \mathrm{We}}{\omega \operatorname{Re}} \sigma_{q}^{i}\right)\right]^{\frac{1}{2}}, \text { for } q>q_{0}, \\
Y_{q}^{i}:=\left[\left\|u_{q}^{i}\right\|_{L^{2}}^{2}+\frac{\mathrm{We}}{\omega \operatorname{Re}}\left\|\Lambda^{-1} \sigma_{q}^{i}\right\|_{L^{2}}^{2}\right]^{\frac{1}{2}}, \text { for } q_{1}<q \leq q_{0},
\end{gathered}
$$

where $q_{0}$ and $q_{1}$ are to be determined later.

Estimates of $Y_{q}^{i}, q \in \mathbb{Z}$.

Step 1: low frequencies: $q \leq q_{1}$.

Taking the $L^{2}$ inner product of $(3.2)_{1}$ and $(3.2)_{2}$ with $u_{q}^{i}$ and $\sigma_{q}^{i}$ respectively, using the divergence free condition $\operatorname{div} u=0$, we obtain

$$
\frac{1}{2} \frac{d}{d t}\left\|u_{q}^{i}\right\|_{L^{2}}^{2}+\frac{1-\omega}{\operatorname{Re}}\left\|\Lambda u_{q}^{i}\right\|_{L^{2}}^{2}-\frac{1}{\operatorname{Re}}\left(u_{q}^{i} \mid \sigma_{q}^{i}\right)=\left(\tilde{f}_{q}^{i} \mid u_{q}^{i}\right)
$$

and

$$
\frac{1}{2} \frac{d}{d t}\left\|\sigma_{q}^{i}\right\|_{L^{2}}^{2}+\frac{1}{\mathrm{We}}\left\|\sigma_{q}^{i}\right\|_{L^{2}}^{2}-\frac{\omega}{\mathrm{We}}\left(\Delta u_{q}^{i} \mid \sigma_{q}^{i}\right)=\left(\tilde{h}_{q}^{i} \mid \sigma_{q}^{i}\right) .
$$

To cancel the "bad" cross term $\left(u_{q}^{i} \mid \sigma_{q}^{i}\right)$ in (3.6), we need the following equality for the $L^{2}$ inner product $\left(u_{q}^{i} \mid \sigma_{q}^{i}\right)$ of $u_{q}^{i}$ and $\sigma_{q}^{i}$,

$$
\frac{d}{d t}\left(u_{q}^{i} \mid \sigma_{q}^{i}\right)+\frac{\omega}{\mathrm{We}}\left\|\Lambda u_{q}^{i}\right\|_{L^{2}}^{2}-\frac{1}{\operatorname{Re}}\left\|\sigma_{q}^{i}\right\|_{L^{2}}^{2}-\frac{1-\omega}{\operatorname{Re}}\left(\Delta u_{q}^{i} \mid \sigma_{q}^{i}\right)+\frac{1}{\mathrm{We}}\left(u_{q}^{i} \mid \sigma_{q}^{i}\right)=\left(\tilde{f}_{q}^{i} \mid \sigma_{q}^{i}\right)+\left(\tilde{h}_{q}^{i} \mid u_{q}^{i}\right),
$$


which is nothing but the sum of $\left((\sqrt{3.2})_{1} \mid \sigma_{q}^{i}\right)$ and $\left((\sqrt{3.2})_{2} \mid u_{q}^{i}\right)$. Multiplying $(\sqrt{3.8})$ by $\frac{\mathrm{We}}{\mathrm{Re}}$, adding the resulting equality to (3.6), one deduces that

$$
\begin{aligned}
\frac{1}{2} \frac{d}{d t}\left(\left\|u_{q}^{i}\right\|_{L^{2}}^{2}+2\left(u_{q}^{i} \mid \frac{\mathrm{We}}{\operatorname{Re}} \sigma_{q}^{i}\right)\right) & +\frac{1}{\operatorname{Re}}\left\|\Lambda u_{q}^{i}\right\|_{L^{2}}^{2}-\frac{\mathrm{We}}{(\operatorname{Re})^{2}}\left\|\sigma_{q}^{i}\right\|_{L^{2}}^{2} \\
& -\frac{1-\omega}{\operatorname{Re}}\left(\Delta u_{q}^{i} \mid \frac{\mathrm{We}}{\operatorname{Re}} \sigma_{q}^{i}\right)=\left(\tilde{f}_{q}^{i} \mid u_{q}^{i}\right)+\frac{\mathrm{We}}{\operatorname{Re}}\left(\left(\tilde{f}_{q}^{i} \mid \sigma_{q}^{i}\right)+\left(\tilde{h}_{q}^{i} \mid u_{q}^{i}\right)\right) .
\end{aligned}
$$

Multiplying (3.7) by $2\left(\frac{\mathrm{We}}{\mathrm{Re}}\right)^{2}$, adding the resulting equality to $(\underline{3.9})$, then the negative term $-\frac{\mathrm{We}}{(\operatorname{Re})^{2}}\left\|\sigma_{q}^{i}\right\|_{L^{2}}^{2}$ in (3.9) is absorbed, and we have

$$
\begin{aligned}
& \frac{1}{2} \frac{d}{d t}\left[\left\|u_{q}^{i}\right\|_{L^{2}}^{2}+2\left\|\frac{\mathrm{We}}{\operatorname{Re}} \sigma_{q}^{i}\right\|_{L^{2}}^{2}+2\left(u_{q}^{i} \mid \frac{\mathrm{We}}{\operatorname{Re}} \sigma_{q}^{i}\right)\right] \\
+ & \frac{1}{\operatorname{Re}}\left\|\Lambda u_{q}^{i}\right\|_{L^{2}}^{2}+\frac{1}{\mathrm{We}}\left\|\frac{\mathrm{We}}{\operatorname{Re}} \sigma_{q}^{i}\right\|_{L^{2}}^{2}-\frac{1+\omega}{\operatorname{Re}}\left(\Delta u_{q}^{i} \mid \frac{\mathrm{We}}{\operatorname{Re}} \sigma_{q}^{i}\right) \\
= & \left(\tilde{f}_{q}^{i} \mid u_{q}^{i}\right)+2\left(\frac{\mathrm{We}}{\operatorname{Re}}\right)^{2}\left(\tilde{h}_{q}^{i} \mid \sigma_{q}^{i}\right)+\frac{\mathrm{We}}{\operatorname{Re}}\left(\left(\tilde{f}_{q}^{i} \mid \sigma_{q}^{i}\right)+\left(\tilde{h}_{q}^{i} \mid u_{q}^{i}\right)\right),
\end{aligned}
$$

In order to exhibit the smoothing effect of $\sigma^{i}, 1 \leq i \leq d$ in the low frequency case, we shall make use of the linear coupling term $-\frac{1}{\mathrm{Re}} \sigma^{i}$ in the first equation of (3.1). To do this, taking the $L^{2}$ inner product of $(3.2)_{1}$ with $\Delta \sigma_{q}^{i}$, and taking the $L^{2}$ inner product of $(3.2)_{2}$ with $\Delta u_{q}^{i}$, then adding them together, we are led to

$$
\begin{aligned}
& \frac{d}{d t}\left(u_{q}^{i} \mid \Delta \sigma_{q}^{i}\right)+\frac{1}{\operatorname{Re}}\left\|\Lambda \sigma_{q}^{i}\right\|_{L^{2}}^{2}-\frac{\omega}{\mathrm{We}}\left\|\Delta u_{q}^{i}\right\|_{L^{2}}^{2}-\frac{1-\omega}{\operatorname{Re}}\left(\Delta u_{q}^{i} \mid \Delta \sigma_{q}^{i}\right)+\frac{1}{\mathrm{We}}\left(\Delta u_{q}^{i} \mid \sigma_{q}^{i}\right) \\
= & \left(\tilde{f}_{q}^{i} \mid \Delta \sigma_{q}^{i}\right)+\left(\tilde{h}_{q}^{i} \mid \Delta u_{q}^{i}\right)-\left(\dot{S}_{q-1} u \cdot \nabla u_{q}^{i} \mid \Delta \sigma_{q}^{i}\right)-\left(\dot{S}_{q-1} u \cdot \nabla \sigma_{q}^{i} \mid \Delta u_{q}^{i}\right) .
\end{aligned}
$$

Multiplying (3.11) by $\frac{\mathrm{We}}{\mathrm{Re}}$, adding the resulting equality to (3.10) yields

$$
\begin{aligned}
& \frac{1}{2} \frac{d}{d t}\left[\left\|u_{q}^{i}\right\|_{L^{2}}^{2}+2\left\|\frac{\mathrm{We}}{\operatorname{Re}} \sigma_{q}^{i}\right\|_{L^{2}}^{2}+2\left(u_{q}^{i} \mid \frac{\mathrm{We}}{\operatorname{Re}} \sigma_{q}^{i}\right)+2\left(u_{q}^{i} \mid \frac{\mathrm{We}}{\operatorname{Re}} \Delta \sigma_{q}^{i}\right)\right] \\
+ & \frac{1}{\operatorname{Re}}\left\|\Lambda u_{q}^{i}\right\|_{L^{2}}^{2}-\frac{\omega}{\operatorname{Re}}\left\|\Delta u_{q}^{i}\right\|_{L^{2}}^{2}+\frac{1}{\mathrm{We}}\left\|\frac{\mathrm{We}}{\operatorname{Re}} \sigma_{q}^{i}\right\|_{L^{2}}^{2}+\frac{1}{\mathrm{We}}\left\|\frac{\mathrm{We}}{\operatorname{Re}} \Lambda \sigma_{q}^{i}\right\|_{L^{2}}^{2} \\
& -\frac{1-\omega}{\mathrm{Re}}\left(\Delta u_{q}^{i} \mid \frac{\mathrm{We}}{\mathrm{Re}} \Delta \sigma_{q}^{i}\right)+\left(\frac{1}{\mathrm{We}}-\frac{1+\omega}{\mathrm{Re}}\right)\left(\Delta u_{q}^{i} \mid \frac{\mathrm{We}}{\mathrm{Re}} \sigma_{q}^{i}\right) \\
= & \left(\tilde{f}_{q}^{i} \mid u_{q}^{i}\right)+2\left(\frac{\mathrm{We}}{\mathrm{Re}}\right)^{2}\left(\tilde{h}_{q}^{i} \mid \sigma_{q}^{i}\right)+\frac{\mathrm{We}}{\mathrm{Re}}\left(\left(\tilde{f}_{q}^{i} \mid \sigma_{q}^{i}\right)+\left(\tilde{h}_{q}^{i} \mid u_{q}^{i}\right)\right) \\
& +\frac{\mathrm{We}}{\operatorname{Re}}\left(\left(\tilde{f}_{q}^{i} \mid \Delta \sigma_{q}^{i}\right)+\left(\tilde{h}_{q}^{i} \mid \Delta u_{q}^{i}\right)-\left(\dot{S}_{q-1} u \cdot \nabla u_{q}^{i} \mid \Delta \sigma_{q}^{i}\right)-\left(\dot{S}_{q-1} u \cdot \nabla \sigma_{q}^{i} \mid \Delta u_{q}^{i}\right)\right),
\end{aligned}
$$

Let us now estimate the cross terms contained in $Y_{q}^{i}$ for $q \leq q_{1}$. Indeed, using Cauchy-Schwarz inequality and (2.4), one easily deduces that

$$
2\left|\left(u_{q}^{i} \mid \frac{\mathrm{We}}{\operatorname{Re}} \sigma_{q}^{i}\right)\right| \leq \frac{5}{8}\left\|u_{q}^{i}\right\|_{L^{2}}^{2}+\frac{8}{5}\left\|\frac{\mathrm{We}}{\operatorname{Re}} \sigma_{q}^{i}\right\|_{L^{2}}^{2},
$$

and

provided

$$
2\left|\left(u_{q}^{i} \mid \frac{\mathrm{We}}{\operatorname{Re}} \Delta \sigma_{q}^{i}\right)\right| \leq\left(\frac{8}{3} 2^{q_{1}}\right)^{2}\left(\left\|u_{q}^{i}\right\|_{L^{2}}^{2}+\left\|\frac{\mathrm{We}}{\operatorname{Re}} \sigma_{q}^{i}\right\|_{L^{2}}^{2}\right) \leq \frac{1}{4}\left(\left\|u_{q}^{i}\right\|_{L^{2}}^{2}+\left\|\frac{\mathrm{We}}{\operatorname{Re}} \sigma_{q}^{i}\right\|_{L^{2}}^{2}\right),
$$

$$
2^{q_{1}} \leq \frac{3}{16}
$$

Consequently,

$$
\left\|u_{q}^{i}\right\|_{L^{2}}^{2}+2\left\|\frac{\mathrm{We}}{\operatorname{Re}} \sigma_{q}^{i}\right\|_{L^{2}}^{2}+2\left(u_{q}^{i} \mid \frac{\mathrm{We}}{\operatorname{Re}} \sigma_{q}^{i}\right)+2\left(u_{q}^{i} \mid \frac{\mathrm{We}}{\operatorname{Re}} \Delta \sigma_{q}^{i}\right) \geq \frac{1}{8}\left\|u_{q}^{i}\right\|_{L^{2}}^{2}+\frac{3}{20}\left\|\frac{\mathrm{We}}{\operatorname{Re}} \sigma_{q}^{i}\right\|_{L^{2}}^{2},
$$


and hence

$$
\left(Y_{q}^{i}\right)^{2} \approx\left\|u_{q}^{i}\right\|_{L^{2}}^{2}+\left\|\frac{\mathrm{We}}{\operatorname{Re}} \sigma_{q}^{i}\right\|_{L^{2}}^{2}, \quad \text { if } \quad q \leq q_{1},
$$

with $q_{1}$ satisfying (3.13). Thanks to (2.4), the remaining two cross terms on the left hand side of (3.12) can be bounded as follows:

$$
\begin{aligned}
\|\left(\frac{1}{\mathrm{We}}-\frac{1+\omega}{\mathrm{Re}}\right)\left(\Delta u_{q}^{i} \mid \frac{\mathrm{We}}{\operatorname{Re}} \sigma_{q}^{i}\right) \mid & \leq 2 \frac{\mathrm{Re}+\mathrm{We}}{\mathrm{WeRe}} \frac{8}{3} 2^{q_{1}}\left\|\Lambda u_{q}^{i}\right\|_{L^{2}}\left\|\frac{\mathrm{We}}{\operatorname{Re}} \sigma_{q}^{i}\right\|_{L^{2}} \\
& \leq \frac{8}{3} 2^{q_{1}} \frac{\operatorname{Re}+\mathrm{We}}{\sqrt{\mathrm{WeRe}}}\left(\frac{1}{\operatorname{Re}}\left\|\Lambda u_{q}^{i}\right\|_{L^{2}}^{2}+\frac{1}{\mathrm{We}}\left\|\frac{\mathrm{We}}{\operatorname{Re}} \sigma_{q}^{i}\right\|_{L^{2}}^{2}\right) \\
& \leq \frac{1}{4}\left(\frac{1}{\operatorname{Re}}\left\|\Lambda u_{q}^{i}\right\|_{L^{2}}^{2}+\frac{1}{\mathrm{We}}\left\|\frac{\mathrm{We}}{\operatorname{Re}} \sigma_{q}^{i}\right\|_{L^{2}}^{2}\right),
\end{aligned}
$$

provided

$$
2^{q_{1}} \leq \frac{3}{32} \frac{\sqrt{\mathrm{WeRe}}}{\mathrm{Re}+\mathrm{We}}
$$

Moreover,

$$
\begin{aligned}
\frac{1-\omega}{\operatorname{Re}}\left|\left(\Delta u_{q}^{i} \mid \frac{\mathrm{We}}{\operatorname{Re}} \Delta \sigma_{q}^{i}\right)\right| & \leq \frac{1}{2}\left(\frac{8}{3} 2^{q_{1}}\right)^{2} \frac{1}{\operatorname{Re}}\left(\left\|\Lambda u_{q}^{i}\right\|_{L^{2}}^{2}+\left\|\frac{\mathrm{We}}{\operatorname{Re}} \Lambda \sigma_{q}^{i}\right\|_{L^{2}}^{2}\right) \\
& \leq \frac{1}{8} \frac{1}{\operatorname{Re}}\left\|\Lambda u_{q}^{i}\right\|_{L^{2}}^{2}+\frac{1}{2} \frac{1}{\mathrm{We}}\left\|\frac{\mathrm{We}}{\operatorname{Re}} \Lambda \sigma_{q}^{i}\right\|_{L^{2}}^{2},
\end{aligned}
$$

provided

$$
2^{q_{1}} \leq \frac{3}{16}, \quad \text { and } \quad 2^{q_{1}} \leq \frac{3}{8} \sqrt{\frac{\mathrm{Re}}{\mathrm{We}}} .
$$

Finally, using (2.4) again yields

$$
\frac{\omega}{\operatorname{Re}}\left\|\Delta u_{q}^{i}\right\|_{L^{2}}^{2} \leq\left(\frac{8}{3} 2^{q^{1}}\right)^{2} \frac{1}{\operatorname{Re}}\left\|\Lambda u_{q}^{i}\right\|_{L^{2}}^{2} \leq \frac{1}{4} \frac{1}{\operatorname{Re}}\left\|\Lambda u_{q}^{i}\right\|_{L^{2}}^{2},
$$

provided (3.13) holds. Now collecting (3.13), (3.16) and (3.18), we take

$$
q_{1}:=\left[\log _{2}\left(\frac{3}{32} \frac{\sqrt{\mathrm{WeRe}}}{\mathrm{Re}+\mathrm{We}}\right)\right] .
$$

From (3.15)-(3.19), and using the left hand side of (2.4), we find that, for $q \leq q_{1}$ with $q_{1}$ satisfying (3.20), there holds

$$
\begin{aligned}
& \frac{1}{\operatorname{Re}}\left\|\Lambda u_{q}^{i}\right\|_{L^{2}}^{2}-\frac{\omega}{\mathrm{Re}}\left\|\Delta u_{q}^{i}\right\|_{L^{2}}^{2}+\frac{1}{\mathrm{We}}\left\|\frac{\mathrm{We}}{\mathrm{Re}} \sigma_{q}^{i}\right\|_{L^{2}}^{2}+\frac{1}{\mathrm{We}}\left\|\frac{\mathrm{We}}{\mathrm{Re}} \Lambda \sigma_{q}^{i}\right\|_{L^{2}}^{2} \\
& -\frac{1-\omega}{\mathrm{Re}}\left(\Delta u_{q}^{i} \mid \frac{\mathrm{We}}{\mathrm{Re}} \Delta \sigma_{q}^{i}\right)+\left(\frac{1}{\mathrm{We}}-\frac{1+\omega}{\mathrm{Re}}\right)\left(\Delta u_{q}^{i} \mid \frac{\mathrm{We}}{\mathrm{Re}} \sigma_{q}^{i}\right) \\
\geq & \frac{3}{8} \frac{1}{\operatorname{Re}}\left\|\Lambda u_{q}^{i}\right\|_{L^{2}}^{2}+\frac{1}{2} \frac{1}{\mathrm{We}}\left\|\frac{\mathrm{We}}{\operatorname{Re}} \Lambda \sigma_{q}^{i}\right\|_{L^{2}}^{2}+\frac{3}{4} \frac{1}{\mathrm{We}}\left\|\frac{\mathrm{We}}{\mathrm{Re}} \sigma_{q}^{i}\right\|_{L^{2}}^{2} \\
\geq & \frac{3}{8}\left(\frac{3}{4} 2^{q}\right)^{2} \min \left\{\frac{1}{\mathrm{We}}, \frac{1}{\mathrm{Re}}\right\}\left(\left\|u_{q}^{i}\right\|_{L^{2}}^{2}+\left\|\frac{\mathrm{We}}{\mathrm{Re}} \sigma_{q}^{i}\right\|_{L^{2}}^{2}\right) .
\end{aligned}
$$

To bound the right hand side of (3.12), firstly integrating by parts and using the divergence free condition $\operatorname{div} u=0$ yields

$$
-\left(\dot{S}_{q-1} u \cdot \nabla u_{q}^{i} \mid \Delta \sigma_{q}^{i}\right)-\left(\dot{S}_{q-1} \cdot \nabla \sigma_{q}^{i} \mid \Delta u_{q}^{i}\right)=\left(\dot{S}_{q-1} \partial_{j} u^{k} \mid \partial_{k} u_{q}^{i} \partial_{j} \sigma_{q}^{i}+\partial_{k} \sigma_{q}^{i} \partial_{j} u_{q}^{i}\right) .
$$


Accordingly, using Bernstein's inequality, (3.20) and Lemma 2.3, we are led to

$$
\begin{aligned}
& \frac{\mathrm{We}}{\operatorname{Re}}\left|\left(\dot{S}_{q-1} u \cdot \nabla u_{q}^{i} \mid \Delta \sigma_{q}^{i}\right)+\left(\dot{S}_{q-1} \cdot \nabla \sigma_{q}^{i} \mid \Delta u_{q}^{i}\right)\right| \\
\leq & C 2^{2 q_{1}}\|\nabla u\|_{L^{\infty}}\left\|u_{q}^{i}\right\|_{L^{2}}\left\|\frac{\mathrm{We}}{\operatorname{Re}} \sigma_{q^{i}}^{i}\right\|_{L^{2}} \leq C\left\|u^{h}\right\|_{\dot{B}_{2,1}^{\frac{d}{2}+1}}^{\frac{1}{2}}\|u\|_{\dot{B}_{2,1}^{\frac{d}{2}+1}}^{\frac{1}{2}}\left(\left\|u_{q}^{i}\right\|_{L^{2}}^{2}+\left\|\frac{\mathrm{We}}{\operatorname{Re}} \sigma_{q^{i}}^{i}\right\|_{L^{2}}^{2}\right),
\end{aligned}
$$

which, together with (3.14), implies that

$$
\text { R.H.S. of (3.12) } \leq C\left(\left\|\tilde{f}_{q}^{i}\right\|_{L^{2}}+\left\|\frac{\mathrm{We}}{\operatorname{Re}} \tilde{h}_{q}^{i}\right\|_{L^{2}}+\left\|u^{h}\right\|_{\dot{B}_{2,1}^{\frac{d}{2}+1}}^{\frac{1}{2}}\|u\|_{\dot{B}_{2,1}^{\frac{d}{2}+1}}^{\frac{1}{2}} Y_{q}^{i}\right) Y_{q}^{i} \text {. }
$$

Substituting (3.23) into (3.12), we infer from (3.14) and (3.21) that there exists a constant $C$ depending on $d$, Re, We, but independent of $\omega$, such that if $q \leq q_{1}$, there holds

$$
\frac{d}{d t}\left(Y_{q}^{i}\right)^{2}+2^{2 q}\left(Y_{q}^{i}\right)^{2} \leq C Y_{q}^{i}\left(\left\|\tilde{f}_{q}^{i}\right\|_{L^{2}}+\left\|\tilde{h}_{q}^{i}\right\|_{L^{2}}+\left\|u^{h}\right\|_{\dot{B}_{2,1}^{\frac{d}{2}+1}}^{\frac{1}{2}}\|u\|_{\dot{B}_{2,1}^{\frac{d}{2}+1}}^{\frac{1}{2}} Y_{q}^{i}\right) .
$$

Step 2: high frequencies: $q>q_{1}$.

Part (i). $q>q_{0}$.

First of all, we would like to point out that, in the high frequency case, the cross term $-\frac{\omega}{\mathrm{We}}\left(\Delta u_{q}^{i} \mid \sigma_{q}^{i}\right)$ in (3.7) is "bad". We will cancel it with the aid of (3.8) once more. To this end, multiplying (3.7) and (3.8) by $\left(\frac{(1-\omega) \mathrm{We}}{\omega \mathrm{Re}}\right)^{2}$ and $-\frac{(1-\omega) \mathrm{We}}{\omega \mathrm{Re}}$ respectively, then adding the resulting equalities together yields

$$
\begin{aligned}
& \frac{1}{2} \frac{d}{d t}\left(\left\|\frac{(1-\omega) \mathrm{We}}{\omega \operatorname{Re}} \sigma_{q}^{i}\right\|_{L^{2}}^{2}-2\left(u_{q}^{i} \mid \frac{(1-\omega) \mathrm{We}}{\omega \operatorname{Re}} \sigma_{q}^{i}\right)\right)-\frac{1-\omega}{\operatorname{Re}}\left\|\Lambda u_{q}^{i}\right\|_{L^{2}}^{2} \\
& +\frac{1}{(1-\omega) \mathrm{We}}\left\|\frac{(1-\omega) \mathrm{We}}{\omega \operatorname{Re}} \sigma_{q}^{i}\right\|_{L^{2}}^{2}-\frac{1}{\mathrm{We}}\left(u_{q}^{i} \mid \frac{(1-\omega) \mathrm{We}}{\omega \operatorname{Re}} \sigma_{q}^{i}\right) \\
= & \left(\frac{(1-\omega) \mathrm{We}}{\omega \operatorname{Re}}\right)^{2}\left(\tilde{h}_{q}^{i} \mid \sigma_{q}^{i}\right)-\frac{(1-\omega) \mathrm{We}}{\omega \operatorname{Re}}\left(\left(\tilde{f}_{q}^{i} \mid \sigma_{q}^{i}\right)+\left(\tilde{h}_{q}^{i} \mid u_{q}^{i}\right)\right) .
\end{aligned}
$$

To absorb the negative term $-\frac{1-\omega}{\operatorname{Re}}\left\|\Lambda u_{q}^{i}\right\|_{L^{2}}^{2}$ in (3.25), we multiply (3.6) by 2 , and add the resulting equality to (3.25) to get

$$
\begin{aligned}
& \frac{1}{2} \frac{d}{d t}\left(2\left\|u_{q}^{i}\right\|_{L^{2}}^{2}+\left\|\frac{(1-\omega) \mathrm{We}}{\omega \operatorname{Re}} \sigma_{q}^{i}\right\|_{L^{2}}^{2}-2\left(u_{q}^{i} \mid \frac{(1-\omega) \mathrm{We}}{\omega \operatorname{Re}} \sigma_{q}^{i}\right)\right)+\frac{1-\omega}{\operatorname{Re}}\left\|\Lambda u_{q}^{i}\right\|_{L^{2}}^{2} \\
& +\frac{1}{(1-\omega) \mathrm{We}}\left\|\frac{(1-\omega) \mathrm{We}}{\omega \operatorname{Re}} \sigma_{q}^{i}\right\|_{L^{2}}^{2}-\frac{2}{\operatorname{Re}}\left(\sigma_{q}^{i} \mid u_{q}^{i}\right)-\frac{1}{\mathrm{We}}\left(u_{q}^{i} \mid \frac{(1-\omega) \mathrm{We}}{\omega \mathrm{Re}} \sigma_{q}^{i}\right) \\
= & 2\left(\tilde{f}_{q}^{i} \mid u_{q}^{i}\right)+\left(\frac{(1-\omega) \mathrm{We}}{\omega \operatorname{Re}}\right)^{2}\left(\tilde{h}_{q}^{i} \mid \sigma_{q}^{i}\right)-\frac{(1-\omega) \mathrm{We}}{\omega \operatorname{Re}}\left(\left(\tilde{f}_{q}^{i} \mid \sigma_{q}^{i}\right)+\left(\tilde{h}_{q}^{i} \mid u_{q}^{i}\right)\right) .
\end{aligned}
$$

It is easy to verify that

$$
2\left\|u_{q}^{i}\right\|_{L^{2}}^{2}+\left\|\frac{(1-\omega) \mathrm{We}}{\omega \mathrm{Re}} \sigma_{q}^{i}\right\|_{L^{2}}^{2}-2\left(u_{q}^{i} \mid \frac{(1-\omega) \mathrm{We}}{\omega \mathrm{Re}} \sigma_{q}^{i}\right) \approx\left\|u_{q}^{i}\right\|_{L^{2}}^{2}+\left\|\frac{(1-\omega) \mathrm{We}}{\omega \mathrm{Re}} \sigma_{q}^{i}\right\|_{L^{2}}^{2} .
$$

Using Cauchy-Schwarz inequality and (2.4) yields

$$
\begin{aligned}
& \left|\frac{2}{\operatorname{Re}}\left(\sigma_{q}^{i} \mid u_{q}^{i}\right)+\frac{1}{\mathrm{We}}\left(u_{q}^{i} \mid \frac{(1-\omega) \mathrm{We}}{\omega \operatorname{Re}} \sigma_{q}^{i}\right)\right| \\
= & \frac{1+\omega}{1-\omega} \frac{1}{\mathrm{We}}\left|\left(u_{q}^{i} \mid \frac{(1-\omega) \mathrm{We}}{\omega \operatorname{Re}} \sigma_{q}^{i}\right)\right| \\
\leq & \frac{1}{2} \frac{1}{1-\omega} \frac{1}{\mathrm{We}}\left\|\frac{(1-\omega) \mathrm{We}}{\omega \operatorname{Re}} \sigma_{q}^{i}\right\|_{L^{2}}^{2}+\frac{2}{(1-\omega) \mathrm{We}}\left\|u_{q}^{i}\right\|_{L^{2}}^{2}
\end{aligned}
$$




$$
\begin{aligned}
& \leq \frac{1}{2} \frac{1}{1-\omega} \frac{1}{\mathrm{We}}\left\|\frac{(1-\omega) \mathrm{We}}{\omega \mathrm{Re}} \sigma_{q}^{i}\right\|_{L^{2}}^{2}+\left(\frac{4}{3} 2^{-q_{0}}\right)^{2} \frac{2}{(1-\omega) \mathrm{We}}\left\|\Lambda u_{q}^{i}\right\|_{L^{2}}^{2} \\
& \leq \frac{1}{2} \frac{1}{1-\omega} \frac{1}{\mathrm{We}}\left\|\frac{(1-\omega) \mathrm{We}}{\omega \mathrm{Re}} \sigma_{q}^{i}\right\|_{L^{2}}^{2}+\frac{1}{2} \frac{1-\omega}{\mathrm{Re}}\left\|\Lambda u_{q}^{i}\right\|_{L^{2}}^{2},
\end{aligned}
$$

provided

$$
\left(\frac{4}{3} 2^{-q_{0}}\right)^{2} \frac{2}{(1-\omega) \mathrm{We}} \leq \frac{1}{2} \frac{1-\omega}{\operatorname{Re}}, \quad \text { i.e. } \quad 2^{q_{0}} \geq \frac{8}{3(1-\omega)} \sqrt{\frac{\mathrm{Re}}{\mathrm{We}}} .
$$

Now let us take

$$
q_{0}:=\left[\log _{2}\left(\frac{8}{3(1-\omega)} \sqrt{\frac{\mathrm{Re}}{\mathrm{We}}}\right)\right]+1 .
$$

Then it follows from (3.28), (3.29) and (2.4) that

$$
\begin{aligned}
& \frac{1-\omega}{\operatorname{Re}}\left\|\Lambda u_{q}^{i}\right\|_{L^{2}}^{2}+\frac{1}{(1-\omega) \mathrm{We}}\left\|\frac{(1-\omega) \mathrm{We}}{\omega \mathrm{Re}} \sigma_{q}^{i}\right\|_{L^{2}}^{2} \\
& -\frac{2}{\operatorname{Re}}\left(\sigma_{q}^{i} \mid u_{q}^{i}\right)-\frac{1}{\mathrm{We}}\left(u_{q}^{i} \mid \frac{(1-\omega) \mathrm{We}}{\omega \mathrm{Re}} \sigma_{q}^{i}\right) \\
\geq & \frac{1}{2} \frac{1-\omega}{\operatorname{Re}}\left\|\Lambda u_{q}^{i}\right\|_{L^{2}}^{2}+\frac{1}{2} \frac{1}{(1-\omega) \mathrm{We}}\left\|\frac{(1-\omega) \mathrm{We}}{\omega \operatorname{Re}} \sigma_{q}^{i}\right\|_{L^{2}}^{2} \\
\geq & \frac{1}{2} \frac{1-\omega}{\operatorname{Re}}\left(\frac{3}{4} 2^{q_{0}}\right)^{2}\left\|u_{q}^{i}\right\|_{L^{2}}^{2}+\frac{1}{2} \frac{1}{(1-\omega) \mathrm{We}}\left\|\frac{(1-\omega) \mathrm{We}}{\omega \operatorname{Re}} \sigma_{q}^{i}\right\|_{L^{2}}^{2} \\
\geq & \frac{1}{2} \frac{1}{(1-\omega) \mathrm{We}}\left(\left\|u_{q}^{i}\right\|_{L^{2}}^{2}+\left\|\frac{(1-\omega) \mathrm{We}}{\omega \operatorname{Re}} \sigma_{q}^{i}\right\|_{L^{2}}^{2}\right) .
\end{aligned}
$$

Finally, the right hand side of (3.26) can be bounded as follows,

$$
\text { R.H.S. of }\left(\underline{3.26)} \leq C\left(\left\|\tilde{f}_{q}^{i}\right\|_{L^{2}}+\left\|\frac{(1-\omega) \mathrm{We}}{\omega \operatorname{Re}} \tilde{h}_{q}^{i}\right\|_{L^{2}}\right)\left(\left\|u_{q}^{i}\right\|_{L^{2}}+\|\left(\frac{1-\omega) \mathrm{We}}{\omega \operatorname{Re}} \sigma_{q}^{i} \|_{L^{2}}\right)\right. \text {. }\right.
$$

Substituting (3.32) into (3.26), using (3.27) and (3.31), we find that there exists a constant $C$ independent of $d$, Re, We, and $\omega$, such that if $q>q_{0}$, there holds

$$
\frac{d}{d t}\left(Y_{q}^{i}\right)^{2}+\frac{1}{(1-\omega) \mathrm{We}}\left(Y_{q}^{i}\right)^{2} \leq C Y_{q}^{i}\left(\left\|\tilde{f}_{q}^{i}\right\|_{L^{2}}+\left\|\frac{(1-\omega) \mathrm{We}}{\omega \operatorname{Re}} \tilde{h}_{q}^{i}\right\|_{L^{2}}\right) .
$$

Part (ii). $q_{1}<q \leq q_{0}$.

From the definitions of $q_{1}$ and $q_{0}$ (see (3.20) and (3.30), it is obvious that there is a gap between the low frequency part $q \leq q_{1}$ and the high frequency part $q>q_{0}$ since $q_{1}<q_{0}$. To overcome this difficulty, it is necessary to resort to some new observation. Considering that $\left\|\Lambda^{-1} \sigma_{q}^{i}\right\|_{L^{2}}$ is equivalent to $\left\|\sigma_{q}^{i}\right\|_{L^{2}}$ if $q_{1}<q \leq q_{0}$, we can use $\left\|\Lambda^{-1} \sigma_{q}^{i}\right\|_{L^{2}}$ instead of $\left\|\sigma_{q}^{i}\right\|_{L^{2}}$ to fill up the gap. To do so, applying the operator $\Lambda^{-1}$ to $(3.2)_{2}$, and then taking the $L^{2}$ inner product of the resulting equation with $\Lambda^{-1} \sigma_{q}^{i}$, we obtain

$$
\begin{aligned}
\frac{1}{2} \frac{d}{d t}\left\|\Lambda^{-1} \sigma_{q}^{i}\right\|_{L^{2}}^{2} & +\frac{1}{\mathrm{We}}\left\|\Lambda^{-1} \sigma_{q}^{i}\right\|_{L^{2}}^{2}+\frac{\omega}{\mathrm{We}}\left(u_{q}^{i} \mid \sigma_{q}^{i}\right) \\
& =\left(\Lambda^{-1} \tilde{h}_{q}^{i} \mid \Lambda^{-1} \sigma_{q}^{i}\right)-\left(\Lambda^{-1}\left(\dot{S}_{q-1} u \cdot \nabla \sigma_{q}^{i}\right) \mid \Lambda^{-1} \sigma_{q}^{i}\right) .
\end{aligned}
$$

Multiplying (3.34) by $\frac{\mathrm{We}}{\omega \mathrm{Re}}$, then adding the resulting equation to (3.6), the cross term $\left(u_{q}^{i} \mid \sigma_{q}^{i}\right)$ is canceled, and we arrive at

$$
\begin{aligned}
& \frac{1}{2} \frac{d}{d t}\left(\left\|u_{q}^{i}\right\|_{L^{2}}^{2}+\frac{\mathrm{We}}{\omega \operatorname{Re}}\left\|\Lambda^{-1} \sigma_{q}^{i}\right\|_{L^{2}}^{2}\right)+\frac{1-\omega}{\operatorname{Re}}\left\|\Lambda u_{q}^{i}\right\|_{L^{2}}^{2}+\frac{1}{\omega \operatorname{Re}}\left\|\Lambda^{-1} \sigma_{q}^{i}\right\|_{L^{2}}^{2} \\
\leq & \left(\tilde{f}_{q}^{i} \mid u_{q}^{i}\right)+\frac{\mathrm{We}}{\omega \operatorname{Re}}\left(\left(\Lambda^{-1} \tilde{h}_{q}^{i} \mid \Lambda^{-1} \sigma_{q}^{i}\right)-\left(\Lambda^{-1}\left(\dot{S}_{q-1} u \cdot \nabla \sigma_{q}^{i}\right) \mid \Lambda^{-1} \sigma_{q}^{i}\right)\right),
\end{aligned}
$$


In view of (2.4) and (3.20), it is not difficult to verify that

$$
\begin{aligned}
\frac{1-\omega}{\operatorname{Re}}\left\|\Lambda u_{q}^{i}\right\|_{L^{2}}^{2}+\frac{1}{\omega \operatorname{Re}}\left\|\Lambda^{-1} \sigma_{q}^{i}\right\|_{L^{2}}^{2} & \geq \frac{1-\omega}{\operatorname{Re}}\left(\frac{3}{4} 2^{q_{1}}\right)^{2}\left\|u_{q}^{i}\right\|_{L^{2}}^{2}+\frac{1}{\omega \operatorname{Re}}\left\|\Lambda^{-1} \sigma_{q}^{i}\right\|_{L^{2}}^{2} \\
& \geq \frac{3^{4}}{2^{16}} \frac{\mathrm{We}(1-\omega)}{(\operatorname{Re}+\mathrm{We})^{2}}\left\|u_{q}^{i}\right\|_{L^{2}}^{2}+\frac{1}{\omega \operatorname{Re}}\left\|\Lambda^{-1} \sigma_{q}^{i}\right\|_{L^{2}}^{2} \\
& \geq \frac{3^{4} \mathrm{We}(1-\omega)}{2^{16}(\operatorname{Re}+\mathrm{We})^{2}}\left(\left\|u_{q}^{i}\right\|_{L^{2}}^{2}+\frac{\mathrm{We}}{\omega \operatorname{Re}}\left\|\Lambda^{-1} \sigma_{q}^{i}\right\|_{L^{2}}^{2}\right) .
\end{aligned}
$$

Using the divergence free condition $\operatorname{div} u=0$, one easily deduces that

$$
\left.\left(\Lambda^{-1}\left(\dot{S}_{q-1} u \cdot \nabla \sigma_{q}^{i}\right) \mid \Lambda^{-1} \sigma_{q}^{i}\right)=\left(\left[\Lambda^{-1}, \dot{S}_{q-1} u \cdot \nabla\right] \sigma_{q}^{i}\right) \mid \Lambda^{-1} \sigma_{q}^{i}\right),
$$

then Lemma 2.2 and Lemma 2.3 imply

$$
\left|\left(\Lambda^{-1}\left(\dot{S}_{q-1} u \cdot \nabla \sigma_{q}^{i}\right) \mid \Lambda^{-1} \sigma_{q}^{i}\right)\right| \leq C\left\|\nabla \dot{S}_{q-1} u\right\|_{L^{\infty}}\left\|\Lambda^{-1} \sigma_{q}^{i}\right\|_{L^{2}}^{2} \leq C\left\|u^{h}\right\|_{\dot{B}_{2,1}^{\frac{d}{2}+1}}^{\frac{1}{2}}\|u\|_{\dot{B}_{2,1}^{\frac{d}{2}+1}}^{\frac{1}{2}}\left\|\Lambda^{-1} \sigma_{q}^{i}\right\|_{L^{2}}^{2} .
$$

Substituting (3.36) and (3.37) into (3.35), we find that there exists a constant $C$ depending on $d$, but independent of $\operatorname{Re}, \mathrm{We}$, and $\omega$, such that if $q_{1}<q \leq q_{0}$, there holds

$$
\frac{d}{d t}\left(Y_{q}^{i}\right)^{2}+\frac{3^{4} \mathrm{We}(1-\omega)}{2^{16}(\mathrm{Re}+\mathrm{We})^{2}}\left(Y_{q}^{i}\right)^{2} \leq C Y_{q}^{i}\left(\left\|\tilde{f}_{q}^{i}\right\|_{L^{2}}+\sqrt{\frac{\mathrm{We}}{\omega \mathrm{Re}}}\left\|\Lambda^{-1} \tilde{h}_{q}^{i}\right\|_{L^{2}}+\left\|u^{h}\right\|_{\dot{B}_{2,1}^{\frac{d}{2}+1}}^{\frac{1}{2}}\|u\|_{\dot{B}_{2,1}^{\frac{d}{2}+1}}^{\frac{1}{2}} Y_{q}^{i}\right)
$$

\section{The smoothing effect of $u$.}

Now we are in a position to show the smoothing effect of the velocity $u$. In fact, in the low frequency part, the smoothing effect of $u_{q}$ has been obtained in (3.24). More precisely, a direct consequence of (3.24) and (3.14) gives

$$
\begin{aligned}
& \left\|u_{q}^{i}(t)\right\|_{L^{2}}+\left\|\sigma_{q}^{i}(t)\right\|_{L^{2}}+2^{2 q}\left(\left\|u_{q}^{i}\right\|_{L_{t}^{1}\left(L^{2}\right)}+\left\|\sigma_{q}^{i}\right\|_{L_{t}^{1}\left(L^{2}\right)}\right) \\
\leq & C\left(\left\|u_{q}^{i}(0)\right\|_{L^{2}}+\left\|\sigma_{q}^{i}(0)\right\|_{L^{2}}+\left\|\tilde{f}_{q}^{i}\right\|_{L_{t}^{1}\left(L^{2}\right)}+\left\|\tilde{h}_{q}^{i}\right\|_{L_{t}^{1}\left(L^{2}\right)}\right. \\
& \left.+\int_{0}^{t}\left\|u^{h}\right\|_{\dot{B}_{2,1}^{\frac{d}{2}+1}}^{\frac{1}{2}}\|u\|_{\dot{B}_{2,1}^{\frac{d}{2}+1}}^{\frac{1}{2}}\left(\left\|u_{q}^{i}\right\|_{L^{2}}+\left\|\sigma_{q}^{i}\right\|_{L^{2}}\right) d t^{\prime}\right),
\end{aligned}
$$

for $q \leq q_{1}$. Nevertheless, in the high frequency part, we infer from (3.33), (3.27) and (3.38) that

$$
\begin{aligned}
& \left\|u_{q}^{i}(t)\right\|_{L^{2}}+\left\|\frac{(1-\omega) \mathrm{We}}{\omega \operatorname{Re}} \sigma_{q}^{i}(t)\right\|_{L^{2}}+\frac{1}{(1-\omega) \mathrm{We}}\left(\left\|u_{q}^{i}\right\|_{L_{t}^{1}\left(L^{2}\right)}+\left\|\frac{(1-\omega) \mathrm{We}}{\omega \operatorname{Re}} \sigma_{q}^{i}\right\|_{L_{t}^{1}\left(L^{2}\right)}\right) \\
\leq & C\left(\left\|u_{q}^{i}(0)\right\|_{L^{2}}+\left\|\frac{(1-\omega) \mathrm{We}}{\omega \operatorname{Re}} \sigma_{q}^{i}(0)\right\|_{L^{2}}+\left\|\tilde{f}_{q}^{i}\right\|_{L_{t}^{1}\left(L^{2}\right)}+\|\left(\frac{(1-\omega) \mathrm{We}}{\omega \operatorname{Re}} \tilde{h}_{q}^{i} \|_{L_{t}^{1}\left(L^{2}\right)}\right),\right.
\end{aligned}
$$

for $q>q_{0}$, and

$$
\begin{aligned}
& \left\|u_{q}^{i}(t)\right\|_{L^{2}}+\sqrt{\frac{\mathrm{We}}{\omega \mathrm{Re}}}\left\|\Lambda^{-1} \sigma_{q}^{i}(t)\right\|_{L^{2}}+\frac{3^{4} \mathrm{We}(1-\omega)}{2^{16}(\mathrm{Re}+\mathrm{We})^{2}}\left(\left\|u_{q}^{i}\right\|_{L_{t}^{1}\left(L^{2}\right)}+\sqrt{\frac{\mathrm{We}}{\omega \mathrm{Re}}}\left\|\Lambda^{-1} \sigma_{q}^{i}\right\|_{L_{t}^{1}\left(L^{2}\right)}\right) \\
\leq & C\left(\left\|u_{q}^{i}(0)\right\|_{L^{2}}+\sqrt{\frac{\mathrm{We}}{\omega \mathrm{Re}}}\left\|\Lambda^{-1} \sigma_{q}^{i}(0)\right\|_{L^{2}}+\left\|\tilde{f}_{q}^{i}\right\|_{L_{t}^{1}\left(L^{2}\right)}+\sqrt{\frac{\mathrm{We}}{\omega \mathrm{Re}}}\left\|\Lambda^{-1} \tilde{h}_{q}^{i}\right\|_{L_{t}^{1}\left(L^{2}\right)}\right. \\
& \left.+\int_{0}^{t}\left\|u^{h}\right\|_{\dot{B}_{2,1}^{\frac{d}{2}+1}}^{\frac{1}{2}}\|u\|_{\dot{B}_{2,1}^{\frac{d}{2}+1}}^{\frac{1}{2}}\left(\left\|u_{q}^{i}\right\|_{L^{2}}+\sqrt{\frac{\mathrm{We}}{\omega \mathrm{Re}}}\left\|\Lambda^{-1} \sigma_{q}^{i}\right\|_{L^{2}}\right) d t^{\prime}\right),
\end{aligned}
$$

for $q_{1}<q \leq q_{0}$. Recalling the definitions of $q_{1}$ and $q_{0}$, and using (2.4) again, one deduces that

$$
\left\|\sigma_{q}^{i}\right\|_{L^{2}} \leq \frac{8}{3} 2^{q_{0}}\left\|\Lambda^{-1} \sigma_{q}^{i}\right\|_{L^{2}} \leq \frac{2^{7}}{3^{2}(1-\omega)} \sqrt{\frac{\mathrm{Re}}{\mathrm{We}}}\left\|\Lambda^{-1} \sigma_{q}^{i}\right\|_{L^{2}}
$$


and

$$
\left\|\sigma_{q}^{i}\right\|_{L^{2}} \geq \frac{3}{4} 2^{q_{1}}\left\|\Lambda^{-1} \sigma_{q}^{i}\right\|_{L^{2}} \geq \frac{3^{2}}{2^{8}} \frac{\sqrt{\mathrm{ReWe}}}{\operatorname{Re}+\mathrm{We}}\left\|\Lambda^{-1} \sigma_{q}^{i}\right\|_{L^{2}} .
$$

By virtue of (3.42), (3.41) implies

$$
\begin{aligned}
& \left\|u_{q}^{i}(t)\right\|_{L^{2}}+\frac{3^{2}(1-\omega) \mathrm{We}}{2^{7} \sqrt{\omega} \operatorname{Re}}\left\|\sigma_{q}^{i}(t)\right\|_{L^{2}} \\
+ & \frac{3^{4} \mathrm{We}(1-\omega)}{2^{16}(\mathrm{Re}+\mathrm{We})^{2}}\left\|u_{q}^{i}\right\|_{L_{t}^{1}\left(L^{2}\right)}+\frac{3^{6}(\mathrm{We})^{2}(1-\omega)^{2}}{\sqrt{\omega} 2^{23}(\mathrm{Re}+\mathrm{We})^{2}} \frac{1}{\operatorname{Re}}\left\|\sigma_{q}^{i}\right\|_{L_{t}^{1}\left(L^{2}\right)} \\
\leq & C\left(\left\|u_{q}^{i}(0)\right\|_{L^{2}}+\sqrt{\frac{\mathrm{We}}{\omega \mathrm{Re}}}\left\|\Lambda^{-1} \sigma_{q}^{i}(0)\right\|_{L^{2}}+\left\|\tilde{f}_{q}^{i}\right\|_{L_{t}^{1}\left(L^{2}\right)}+\sqrt{\frac{\mathrm{We}}{\omega \mathrm{Re}}}\left\|\Lambda^{-1} \tilde{h}_{q}^{i}\right\|_{L_{t}^{1}\left(L^{2}\right)}\right. \\
& \left.+\int_{0}^{t}\left\|u^{h}\right\|_{\dot{B}_{2,1}^{\frac{d}{2}+1}}^{\frac{1}{2}}\|u\|^{\frac{1}{2}}\left(\left\|u_{\dot{B}_{2,1}^{\frac{d}{2}+1}}^{i}\right\|_{L^{2}}+\sqrt{\frac{\mathrm{We}}{\omega \mathrm{Re}}}\left\|\Lambda^{-1} \sigma_{q}^{i}\right\|_{L^{2}}\right) d t^{\prime}\right),
\end{aligned}
$$

for $q_{1}<q \leq q_{0}$. It can be seen from (3.40) and (3.44) that we have not yet obtain the soothing effect of $u_{q}$ if $q>q_{1}$. Fortunately, the damping effect of $\sigma_{q}$ is obtained both in (3.40) and (3.44). This enables us to get the smoothing effect of $u_{q}$ by means of the equation of the velocity $u$. To this end, taking the $L^{2}$ inner product of $(3.2)_{1}$ with $u_{q}^{i}$, integrating by parts, we have

$$
\frac{1}{2} \frac{d}{d t}\left\|u_{q}^{i}\right\|_{L^{2}}^{2}+\frac{1-\omega}{\operatorname{Re}}\left\|\Lambda u_{q}^{i}\right\|_{L^{2}}^{2}=\frac{1}{\operatorname{Re}}\left(\sigma_{q}^{i} \mid u_{q}^{i}\right)+\left(\tilde{f}_{q}^{i} \mid u_{q}^{i}\right) .
$$

Using the left hand side of (2.4), we easily get

$$
\left\|u_{q}^{i}(t)\right\|_{L^{2}}+\frac{1-\omega}{\operatorname{Re}}\left(\frac{3}{4} 2^{q}\right)^{2}\left\|u_{q}^{i}\right\|_{L_{t}^{1}\left(L^{2}\right)} \leq\left\|u_{q}^{i}(0)\right\|_{L^{2}}+\frac{1}{\operatorname{Re}}\left\|\sigma_{q}^{i}\right\|_{L_{t}^{1}\left(L^{2}\right)}+\left\|\tilde{f}_{q}^{i}\right\|_{L_{t}^{1}\left(L^{2}\right)} .
$$

Multiplying (3.40) by $2 \omega$, and adding the resulting equation to (3.45), we arrive at

$$
\begin{aligned}
& \left\|u_{q}^{i}(t)\right\|_{L^{2}}+\frac{2(1-\omega) \mathrm{We}}{\operatorname{Re}}\left\|\sigma_{q}^{i}(t)\right\|_{L^{2}}+\frac{1-\omega}{\operatorname{Re}}\left(\frac{3}{4} 2^{q}\right)^{2}\left\|u_{q}^{i}\right\|_{L_{t}^{1}\left(L^{2}\right)}+\frac{1}{\operatorname{Re}}\left\|\sigma_{q}^{i}\right\|_{L_{t}^{1}\left(L^{2}\right)} \\
\leq & C\left(\left\|u_{q}^{i}(0)\right\|_{L^{2}}+\left\|\sigma_{q}^{i}(0)\right\|_{L^{2}}+\left\|\tilde{f}_{q}^{i}\right\|_{L_{t}^{1}\left(L^{2}\right)}+\left\|\tilde{h}_{q}^{i}\right\|_{L_{t}^{1}\left(L^{2}\right)}\right),
\end{aligned}
$$

for $q>q_{0}$. Multiplying (3.44) by $\frac{\sqrt{\omega} 2^{24}(\mathrm{Re}+\mathrm{We})^{2}}{3^{6}(\mathrm{We})^{2}(1-\omega)^{2}}$, and adding the resulting equation to (3.45), using (3.43), we obtain

$$
\begin{aligned}
& \left\|u_{q}^{i}(t)\right\|_{L^{2}}+\frac{2^{17}(\operatorname{Re}+\mathrm{We})^{2}}{3^{4} \operatorname{ReWe}(1-\omega)}\left\|\sigma_{q}^{i}(t)\right\|_{L^{2}}+\frac{1-\omega}{\operatorname{Re}}\left(\frac{3}{4} 2^{q}\right)^{2}\left\|u_{q}^{i}\right\|_{L_{t}^{1}\left(L^{2}\right)}+\frac{1}{\operatorname{Re}}\left\|\sigma_{q}^{i}\right\|_{L_{t}^{1}\left(L^{2}\right)} \\
\leq & \frac{C}{(1-\omega)^{2}}\left(\left\|u_{q}^{i}(0)\right\|_{L^{2}}+\left\|\sigma_{q}^{i}(0)\right\|_{L^{2}}+\left\|\tilde{f}_{q}^{i}\right\|_{L_{t}^{1}\left(L^{2}\right)}\right. \\
& \left.+\left\|\tilde{h}_{q}^{i}\right\|_{L_{t}^{1}\left(L^{2}\right)}+\int_{0}^{t}\left\|u^{h}\right\|_{\dot{B}_{2,1}^{\frac{d}{2}+1}}^{\frac{1}{2}}\|u\|_{\dot{B}_{2,1}^{\frac{d}{2}+1}}^{\frac{1}{2}}\left(\left\|u_{q}^{i}\right\|_{L^{2}}+\left\|\sigma_{q}^{i}\right\|_{L^{2}}\right) d t^{\prime}\right),
\end{aligned}
$$

for $q_{1}<q \leq q_{0}$. Now collecting (3.46), (3.47), and (3.39), we conclude that

$$
\begin{aligned}
& \left\|u_{\tilde{L}_{t}^{\infty} \|_{2,1}^{\dot{B}_{2}} \dot{d}^{2}-1}+(1-\omega)\right\| \sigma^{i}\left\|_{\tilde{L}_{t}^{\infty}\left(\dot{B}_{2,1}^{\frac{d}{2}-1}\right)}+(1-\omega)\right\| u_{L_{t}^{i} \|_{\left.\dot{B}_{2,1}^{\frac{d}{2}+1}\right)}}+\left\|\sigma^{i}\right\|_{L_{t}^{1}\left(\dot{B}_{2,1}^{\frac{d}{2}+1, \frac{d}{2}-1}\right)} \\
\leq & \frac{C}{(1-\omega)^{2}}\left(\left\|u_{0}^{i}\right\|_{\dot{B}_{2,1}^{\frac{d}{2}-1}}+\left\|\sigma_{0}^{i}\right\|_{\dot{B}_{2,1}^{\frac{d}{2}-1}}+\int_{0}^{t} \sum_{q \in \mathbb{Z}} 2^{q\left(\frac{d}{2}-1\right)}\left(\left\|\tilde{f}_{q}^{i}\right\|_{L^{2}}+\left\|\tilde{h}_{q}^{i}\right\|_{L^{2}}\right) d t^{\prime}\right. \\
& \left.+\int_{0}^{t}\left\|u^{h}\right\|_{\dot{B}_{2,1}^{\frac{d}{2}+1}}^{\frac{1}{2}}\|u\|_{\dot{B}_{2,1}^{\frac{d}{2}+1}}^{\frac{1}{2}}\left(\left\|u^{i}\right\|_{\dot{B}_{2,1}^{\frac{d}{2}-1}}+\left\|\sigma^{i}\right\|_{\dot{B}_{2,1}^{\frac{d}{2}-1}}\right) d t^{\prime}\right)
\end{aligned}
$$


with some positive constant $C$ depending on $d$, Re and We, but independent of $\omega$.

Estimates of $\sum_{q \in \mathbb{Z}} 2^{q\left(\frac{d}{2}-1\right)}\left(\left\|\tilde{f}_{q}^{i}\right\|_{L^{2}}+\left\|\tilde{h}_{q}^{i}\right\|_{L^{2}}\right)$.

Recalling that $\tilde{f}_{q}^{i}:=\dot{S}_{q-1} u \cdot \nabla u_{q}^{i}+f_{q}^{i}$, and $f^{i}:=-(\mathbb{P}(u \cdot \nabla) u)^{i}=-\left(\delta_{i m}+\partial_{i} \Lambda^{-2} \partial_{m}\right) u^{k} \partial_{k} u^{m}$, in order to estimate $\sum_{q \in \mathbb{Z}} 2^{q\left(\frac{d}{2}-1\right)}\left\|\tilde{f}_{q}^{i}\right\|_{L^{2}}$, it suffices to bound $\sum_{q \in \mathbb{Z}} 2^{\left(\frac{d}{2}-1\right)}\left\|\dot{S}_{q-1} u^{k} \partial_{k} u_{q}^{i}\right\|_{L^{2}}$ and $\|\left(\delta_{i m}+\right.$ $\left.\partial_{i} \Lambda^{-2} \partial_{m}\right) u^{k} \partial_{k} u^{m} \|_{\dot{B}_{2,1}^{\frac{d}{2}-1}}$.

Estimates of $\sum_{q \in \mathbb{Z}} 2^{\left(\frac{d}{2}-1\right)}\left\|\dot{S}_{q-1} u^{k} \partial_{k} u_{q}^{i}\right\|_{L^{2}}$.

- Case 1: $1 \leq i \leq d-1$.

$$
\begin{aligned}
\sum_{q \in \mathbb{Z}} 2^{\left(\frac{d}{2}-1\right)}\left\|\dot{S}_{q-1} u^{k} \partial_{k} u_{q}^{i}\right\|_{L^{2}} & \leq C\left\|\dot{S}_{q-1} u\right\|_{L^{\infty}} \sum_{q \in \mathbb{Z}} 2^{\frac{d}{2}}\left\|u_{q}^{h}\right\|_{L^{2}} \\
& \leq C\|u\|_{L^{\infty}}\left\|u^{h}\right\|_{\dot{B}_{2,1}^{\frac{d}{2}}} \leq C\left(\left\|u^{h}\right\|_{\dot{B}_{2,1}^{\frac{d}{2}}}^{2}+\left\|u^{h}\right\|_{\dot{B}_{2,1}^{\frac{d}{2}}}\left\|u^{d}\right\|_{\dot{B}_{2,1}^{\frac{d}{2}}}\right) .
\end{aligned}
$$

- Case 2: $i=d$.

$$
\begin{aligned}
\sum_{q \in \mathbb{Z}} 2^{\left(\frac{d}{2}-1\right)}\left\|\dot{S}_{q-1} u^{k} \partial_{k} u_{q}^{d}\right\|_{L^{2}} & \leq C \sum_{q \in \mathbb{Z}} 2^{\left(\frac{d}{2}-1\right)}\left\|\dot{S}_{q-1} u^{h} \partial_{h} u_{q}^{d}\right\|_{L^{2}}+\sum_{q \in \mathbb{Z}} 2^{\frac{d}{2}-1}\left\|\dot{S}_{q-1} u^{d} \partial_{d} u_{q}^{d}\right\|_{L^{2}} \\
& \leq C\left\|u^{h}\right\|_{L^{\infty}}\left\|u^{d}\right\|_{\dot{B}_{2,1}^{\frac{d}{2}}}+C\left\|u^{d}\right\|_{L^{\infty}}\left\|\operatorname{div}_{h} u^{h}\right\|_{\dot{B}_{2,1}^{\frac{d}{2}-1}} \\
& \leq C\left\|u^{h}\right\|_{\dot{B}_{2,1}^{\frac{d}{2}}}\left\|u^{d}\right\|_{\dot{B}_{2,1}^{\frac{d}{2}}} .
\end{aligned}
$$

Combining these two estimates, we are led to

$$
\sum_{q \in \mathbb{Z}} 2^{\left(\frac{d}{2}-1\right)}\left\|\dot{S}_{q-1} u^{k} \partial_{k} u_{q}^{i}\right\|_{L^{2}} \leq C\left(\left\|u^{h}\right\|_{\dot{B}_{2,1}^{\frac{d}{2}}}^{2}+\left\|u^{h}\right\|_{\dot{B}_{2,1}^{\frac{d}{2}}}\left\|u^{d}\right\|_{\dot{B}_{2,1}^{\frac{d}{2}}}\right) \quad \text { for } \quad 1 \leq i \leq d .
$$

Estimates of $\left\|\left(\delta_{i m}+\partial_{i} \Lambda^{-2} \partial_{m}\right) u^{k} \partial_{k} u^{m}\right\|_{\dot{B}_{2,1}^{\frac{d}{2}-1}}$.

Using Proposition 2.2, we have

$$
\begin{aligned}
& \left\|\left(\delta_{i m}+\partial_{i} \Lambda^{-2} \partial_{m}\right) \dot{T}_{u^{k}} \partial_{k} u^{m}\right\|_{\dot{B}_{2,1}^{\frac{d}{2}-1}} \\
\leq & \sum_{1 \leq m, k \leq d-1}\left\|\left(\delta_{i m}+\partial_{i} \Lambda^{-2} \partial_{m}\right) \dot{T}_{u^{k}} \partial_{k} u^{m}\right\|_{\dot{B}_{2,1}^{\frac{d}{2}-1}}+\sum_{1 \leq k \leq d-1}\left\|\left(\delta_{i d}+\partial_{i} \Lambda^{-2} \partial_{d}\right) \dot{T}_{u^{k}} \partial_{k} u^{d}\right\|_{\dot{B}_{2,1}^{\frac{d}{2}-1}} \\
& +\sum_{1 \leq m \leq d-1}\left\|\left(\delta_{i m}+\partial_{i} \Lambda^{-2} \partial_{m}\right) \dot{T}_{u^{d}} \partial_{d} u^{m}\right\|_{\dot{B}_{2,1}^{\frac{d}{2}-1}}+\left\|\left(\delta_{i d}+\partial_{i} \Lambda^{-2} \partial_{d}\right) \dot{T}_{u^{d}} \partial_{d} u^{d}\right\|_{\dot{B}_{2,1}^{2}-1} \\
\leq & C\left(\left\|u^{h}\right\|_{L^{\infty}}\left\|\partial_{h} u^{h}\right\|_{\dot{B}_{2,1}^{\frac{d}{2}-1}}+\left\|u^{h}\right\|_{L^{\infty}}\left\|\partial_{h} u^{d}\right\|_{\dot{B}_{2,1}^{\frac{d}{2}-1}}+\left\|u^{d}\right\|_{L^{\infty}}\left\|\partial_{d} u^{h}\right\|_{\dot{B}_{2,1}^{\frac{d}{2}-1}}+\left\|u^{d}\right\|_{L^{\infty}}\left\|\operatorname{div}_{h} u^{h}\right\|_{\dot{B}_{2,1}^{\frac{d}{2}-1}}\right) \\
\leq & C\left(\left\|u^{h}\right\|_{\dot{B}_{2,1}^{\frac{d}{2}}}^{2}+\left\|u^{h}\right\|_{\dot{B}_{2,1}^{\frac{d}{2}}}\left\|u^{d}\right\|_{\dot{B}_{2,1}^{\frac{d}{2}}}\right),
\end{aligned}
$$

and

$$
\begin{aligned}
& \left\|\left(\delta_{i m}+\partial_{i} \Lambda^{-2} \partial_{m}\right) \dot{T}_{\partial_{k} u^{m}}^{\prime} u^{k}\right\|_{\dot{B}_{2,1}^{\frac{d}{2}-1}}^{2} \\
\leq & \sum_{1 \leq m, k \leq d-1}\left\|\left(\delta_{i m}+\partial_{i} \Lambda^{-2} \partial_{m}\right) \dot{T}_{\partial_{k} u^{m}}^{\prime} u^{k}\right\|_{\dot{B}_{2,1}^{\frac{d}{2}-1}}+\sum_{1 \leq k \leq d-1}\left\|\left(\delta_{i d}+\partial_{i} \Lambda^{-2} \partial_{d}\right) \dot{T}_{\partial_{k} u^{d}}^{\prime} u^{k}\right\|_{\dot{B}_{2,1}^{\frac{d}{2}-1}} \\
& +\sum_{1 \leq m \leq d-1}\left\|\left(\delta_{i m}+\partial_{i} \Lambda^{-2} \partial_{m}\right) \dot{T}_{\partial_{d} u^{m}}^{\prime} u^{d}\right\|_{\dot{B}_{2,1}^{\frac{d}{2}-1}}+\left\|\left(\delta_{i d}+\partial_{i} \Lambda^{-2} \partial_{d}\right) \dot{T}_{\partial_{d} u^{d}}^{\prime} u^{d}\right\|_{\dot{B}_{2,1}^{2 \frac{d}{2}-1}}
\end{aligned}
$$




$$
\begin{aligned}
& \leq C\left(\left\|\partial_{h} u^{h}\right\|_{\dot{B}_{2,1}^{\frac{d}{2}}}\left\|u^{h}\right\|_{\dot{B}_{2,1}^{\frac{d}{2}-1}}+\left\|\partial_{h} u^{d}\right\|_{\dot{B}_{2,1}^{\frac{d}{2}-1}}\left\|u^{h}\right\|_{\dot{B}_{2,1}^{\frac{d}{2}}}+\left\|\partial_{d} u^{h}\right\|_{\dot{B}_{2,1}^{\frac{d}{2}-1}}\left\|u^{d}\right\|_{\dot{B}_{2,1}^{\frac{d}{2}}}+\left\|\operatorname{div}_{h} u^{h}\right\|_{\dot{B}_{2,1}^{\frac{d}{2}-1}}\left\|u^{d}\right\|_{\dot{B}_{2,1}^{\frac{d}{2}}}\right) \\
& \leq C\left(\left\|u^{h}\right\|_{\dot{B}_{2,1}^{\frac{d}{2}-1}}\left\|u^{h}\right\|_{\dot{B}_{2,1}^{\frac{d}{2}+1}}+\left\|u^{h}\right\|_{\dot{B}_{2,1}^{\frac{d}{2}}}\left\|u^{d}\right\|_{\dot{B}_{2,1}^{\frac{d}{2}}}\right) .
\end{aligned}
$$

Therefore, according to Bony's decomposition, there holds

$$
\left\|\left(\delta_{i m}+\partial_{i} \Lambda^{-2} \partial_{m}\right) u^{k} \partial_{k} u^{m}\right\|_{\dot{B}_{2,1}^{\frac{d}{2}-1}} \leq C\left(\left\|u^{h}\right\|_{\dot{B}_{2,1}^{\frac{d}{2}-1}}\left\|u^{h}\right\|_{\dot{B}_{2,1}^{\frac{d}{2}+1}}+\left\|u^{h}\right\|_{\dot{B}_{2,1}^{\frac{d}{2}}}\left\|u^{d}\right\|_{\dot{B}_{2,1}^{\frac{d}{2}}}\right) \quad \text { for } \quad 1 \leq i \leq d .
$$

It follows from (3.49), (3.50) and Proposition 2.1 that

$$
\begin{aligned}
& \sum_{q \in \mathbb{Z}} 2^{q\left(\frac{d}{2}-1\right)}\left\|\tilde{f}_{q}^{i}\right\|_{L^{2}} \leq C\left(\left\|u^{h}\right\|_{\dot{B}_{2,1}^{\frac{d}{2}-1}}\left\|u^{h}\right\|_{\dot{B}_{2,1}^{\frac{d}{2}+1}}+\left\|u^{h}\right\|_{\dot{B}_{2,1}^{\frac{d}{2}}}\left\|u^{d}\right\|_{\dot{B}_{2,1}^{\frac{d}{2}}}\right) \\
& \leq C\left(\left\|u^{h}\right\|_{\dot{B}_{2,1}^{\frac{d}{2}-1}}\left\|u^{h}\right\|_{\dot{B}_{2,1}^{\frac{d}{2}+1}}+\left\|u^{h}\right\|_{\dot{B}_{2,1}^{\frac{d}{2}+1}}^{\frac{1}{2}}\left\|u^{d}\right\|_{\dot{B}_{2,1}^{\frac{d}{2}-1}}^{\frac{1}{2}}\left\|u^{h}\right\|_{\dot{B}_{2,1}^{\frac{d}{2}-1}}^{\frac{1}{2}}\left\|u^{d}\right\|_{\dot{B}_{2,1}^{\frac{d}{2}+1}}^{\frac{1}{2}}\right) \quad \text { for } \quad 1 \leq i \leq d .
\end{aligned}
$$

Next, we turn to estimate $\sum_{q \in \mathbb{Z}} 2^{q\left(\frac{d}{2}-1\right)}\left\|\tilde{h}_{q}^{i}\right\|_{L^{2}}$. Noticing that

$$
\begin{aligned}
\tilde{h}_{q}^{i}= & \dot{S}_{q-1} u \cdot \nabla \sigma_{q}^{i}+h_{q}^{i} \\
= & \dot{S}_{q-1} u \cdot \nabla \sigma_{q}^{i}-\dot{\Delta}_{q}\left(\mathbb{P} \operatorname{div}(u \cdot \nabla) \tau+\mathbb{P} \operatorname{div} g_{\alpha}(\tau, \nabla u)\right)^{i} \\
= & {\left[\dot{S}_{q-1} u \cdot \nabla \sigma_{q}^{i}-\dot{\Delta}_{q}\left(\mathbb{P} \dot{T}_{u^{k}} \partial_{k} \operatorname{div} \tau\right)^{i}\right]-\dot{\Delta}_{q}\left(\delta_{i m}+\partial_{i} \Lambda^{-2} \partial_{m}\right) \dot{T}_{\partial_{l} u^{k}} \partial_{k} \tau^{l, m} } \\
& -\dot{\Delta}_{q}\left(\delta_{i m}+\partial_{i} \Lambda^{-2} \partial_{m}\right) \partial_{l} \dot{T}_{\partial_{k} \tau^{l, m}}^{\prime} u^{k}-\dot{\Delta}_{q}\left(\mathbb{P} \operatorname{div} g_{\alpha}(\tau, \nabla u)\right)^{i},
\end{aligned}
$$

we bound $\sum_{q \in \mathbb{Z}} 2^{q\left(\frac{d}{2}-1\right)}\left\|\tilde{h}_{q}^{i}\right\|_{L^{2}}$ term by term. First of all, thanks to the commutate estimate Lemma 10.25 in [1], we find that

$$
\begin{aligned}
& \sum_{q \in \mathbb{Z}} 2^{q\left(\frac{d}{2}-1\right)}\left\|\left[\dot{S}_{q-1} u \cdot \nabla \sigma_{q}^{i}-\dot{\Delta}_{q}\left(\mathbb{P} \dot{T}_{u^{k}} \partial_{k} \operatorname{div} \tau\right)^{i}\right]\right\|_{L^{2}} \\
\leq & C\|\nabla u\|_{L^{\infty}} \sum_{q \in \mathbb{Z}} \sum_{\left|q^{\prime}-q\right| \leq 4} 2^{q\left(\frac{d}{2}-1\right)}\left\|\dot{\Delta}_{q^{\prime}} \operatorname{div} \tau\right\|_{L^{2}} \leq C \| u_{\dot{B}_{2,1}^{h}\left\|_{\dot{B}_{2,1}^{\frac{d}{2}+1}}^{\frac{1}{2}}\right\| u\left\|_{\dot{B}_{2,1}^{\frac{d}{2}+1}}^{\frac{1}{2}}\right\| \tau \|_{\dot{B}_{2}^{\frac{d}{2}}}} .
\end{aligned}
$$

The second term contained in $\sum_{q \in \mathbb{Z}} 2^{q\left(\frac{d}{2}-1\right)}\left\|\tilde{h}_{q}^{i}\right\|_{L^{2}}$ is nothing but $\left\|\left(\delta_{i m}+\partial_{i} \Lambda^{-2} \partial_{m}\right) \dot{T}_{\partial_{l} l^{k}} \partial_{k} \tau^{l, m}\right\|_{\dot{B}_{2,1}^{\frac{d}{2}-1}}$, the estimate of which is easier. Indeed, using Proposition 2.1 and 2.2 directly yields

$$
\left\|\left(\delta_{i m}+\partial_{i} \Lambda^{-2} \partial_{m}\right) \dot{T}_{\partial_{l} u^{k}} \partial_{k} \tau^{l, m}\right\|_{\dot{B}_{2,1}^{\frac{d}{2}-1}} \leq C\|\nabla u\|_{L^{\infty}}\|\nabla \tau\|_{\dot{B}_{2,1}^{\frac{d}{2}-1}} \leq C\left\|u_{\dot{B}_{2,1}^{h} \|^{\frac{d}{2}+1}}^{\frac{1}{2}}\right\| u\left\|_{\dot{B}_{2,1}^{\frac{d}{2}+1}}^{\frac{1}{2}}\right\| \tau \|_{\dot{B}_{2,1}^{\frac{d}{2}}} \cdot
$$

Now we go to bound the third term,

$$
\left\|\left(\delta_{i m}+\partial_{i} \Lambda^{-2} \partial_{m}\right) \partial_{l} \dot{T}_{\partial_{k} \tau}^{\prime} \tau^{i, m} u^{k}\right\|_{\dot{B}_{2,1}^{\frac{d}{2}-1}} \leq C\left\|\dot{T}_{\partial_{k} \tau}^{\prime} u^{k}\right\|_{\dot{B}_{2,1}^{\frac{d}{2}}} \leq C\left\|\dot{T}_{\partial_{h} \tau}^{\prime} u^{h}\right\|_{\dot{B}_{2,1}^{\frac{d}{2}}}+C\left\|\dot{T}_{\partial_{d} \tau}^{\prime} u^{d}\right\|_{\dot{B}_{2,1}^{\frac{d}{2}}} \cdot
$$

Using Proposition 2.2 once more and Bernstein's inequality, it is easy to see that

$$
\left\|\dot{T}_{\partial_{h} \tau}^{\prime} u^{h}\right\|_{\dot{B}_{2,1}^{\frac{d}{2}}} \leq C\left\|\partial_{h} \tau\right\|_{\dot{B}_{\infty, \infty}^{-1}}\left\|u^{h}\right\|_{\dot{B}_{2,1}^{\frac{d}{2}+1}} \leq C\left\|u_{\dot{B}_{2,1}^{h}}\right\|_{\dot{B}_{2,1}^{2}}\|\tau\|^{\frac{d}{2}}
$$

However, the estimate of $\left\|\dot{T}_{\partial_{d} \tau}^{\prime} u^{d}\right\|_{\dot{B}_{2,1}^{\frac{d}{2}}}$ is a little bit complicated. In fact, using Lemma 2.3 and Bernstein's inequality, we have

$$
\begin{aligned}
\left\|\dot{T}_{\partial_{d} \tau}^{\prime} u^{d}\right\|_{\dot{B}_{2,1}^{\frac{d}{2}}} & =\sum_{q \in Z} 2^{q \frac{d}{2}}\left\|\dot{\Delta}_{q} \dot{T}_{\partial_{d} \tau}^{\prime} u^{d}\right\|_{L^{2}} \\
& \leq \sum_{q \in Z} \sum_{q-q^{\prime} \lesssim 1} 2^{q \frac{d}{2}}\left\|\dot{\Delta}_{q}\left(\dot{S}_{q^{\prime}+2} \partial_{d} \tau \dot{\Delta}_{q^{\prime}} u^{d}\right)\right\|_{L^{2}}
\end{aligned}
$$




$$
\begin{aligned}
& \leq \sum_{q \in Z} \sum_{q^{\prime} q^{\prime} \lesssim 1} 2^{q \frac{d}{2}}\left\|\dot{S}_{q^{\prime}+2} \partial_{d} \tau\right\|_{L_{x_{h}}^{\infty}\left(L_{x_{d}}^{2}\right)}\left\|\dot{\Delta}_{q^{\prime}} u^{d}\right\|_{L_{x_{h}}^{2}\left(L_{x_{d}}^{\infty}\right)} \\
& \leq C \sum_{q \in Z} \sum_{q-q^{\prime} \lesssim 1} \sum_{q^{\prime \prime} \leq q^{\prime}+1} 2^{q \frac{d}{2}}\left\|\dot{\Delta}_{q^{\prime \prime}} \partial_{d} \tau\right\|_{L_{x_{h}}^{\infty}\left(L_{x_{d}}^{2}\right.}\left\|\dot{\Delta}_{q^{\prime}} u^{d}\right\|_{L^{2}}^{\frac{1}{2}}\left\|\dot{\Delta}_{q^{\prime}} \operatorname{div}_{h} u^{h}\right\|_{L^{2}}^{\frac{1}{2}} \\
& \leq C \sum_{q \in Z} \sum_{q-q^{\prime} \lesssim 1} \sum_{q^{\prime \prime} \leq q^{\prime}+1} 2^{q \frac{d}{2}} 2^{q^{\prime \prime}} \frac{d+1}{2}\left\|\dot{\Delta}_{q^{\prime \prime}} \tau\right\|_{L^{2}}\left\|\dot{\Delta}_{q^{\prime}} u^{d}\right\|_{L^{2}}^{\frac{1}{2}}\left\|\dot{\Delta}_{q^{\prime}} \operatorname{div}_{h} u^{h}\right\|_{L^{2}}^{\frac{1}{2}} \\
& \leq C \sum_{q \in Z} \sum_{q-q^{\prime} \lesssim 1} 2^{\left(q-q^{\prime}\right) \frac{d}{2}} \sum_{q^{\prime \prime} \leq q^{\prime}+1} 2^{\left(q^{\prime \prime}-q^{\prime}\right) \frac{1}{2}}\left(2^{q^{\prime \prime} \frac{d}{2}}\left\|\dot{\Delta}_{q^{\prime \prime}} \tau\right\|_{L^{2}}\right) \\
& \quad \times\left(2^{q^{\prime}\left(\frac{d}{2}+1\right)}\left\|\dot{\Delta}_{q^{\prime}} u^{d}\right\|_{L^{2}}\right)^{\frac{1}{2}}\left(2^{q^{\prime} \frac{d}{2}}\left\|\dot{\Delta}_{q^{\prime}} \operatorname{div}_{h} u^{h}\right\|_{L^{2}}\right)^{\frac{1}{2}} \\
& \leq C\left\|u^{d}\right\|_{\dot{B}_{2,1}^{\frac{1}{2}+1}}^{\frac{1}{2}}\left\|u^{h}\right\|_{\dot{B}_{2,1}^{\frac{d}{2}+1}}^{\frac{1}{2}}\|\tau\|_{\dot{B}_{2,1}^{\frac{d}{2}}} \cdot
\end{aligned}
$$

Finally, we would like to point out that the estimate of the term $\left\|\left(\mathbb{P} \operatorname{div} g_{\alpha}(\tau, \nabla u)\right)^{i}\right\|_{\dot{B}_{2,1}^{2}-1}$ is somehow "bad" due to the appearance of $\sum_{1 \leq k \leq d-1}\left\|\dot{T}_{\tau^{l, k}} \partial_{k} u^{d}\right\|_{\dot{B}_{2,1}^{\frac{d}{2}}}$ both for horizontal and vertical direction. Thus, there is no need to bound $\left\|\left(\mathbb{P} \operatorname{div} g_{\alpha}(\tau, \nabla u)\right)^{i}\right\|_{\dot{B}_{2,1}^{\frac{d}{2}-1}}$ from different directions. In view of Proposition 2.1 and Corollary 2.2 , we easily get

$$
\begin{aligned}
\left\|\left(\mathbb{P} \operatorname{div} g_{\alpha}(\tau, \nabla u)\right)^{i}\right\|_{\dot{B}_{2,1}^{\frac{d}{2}-1}} & \leq C\left\|g_{\alpha}(\tau, \nabla u)\right\|_{\dot{B}_{2,1}^{\frac{d}{2}}} \\
& \leq C\|\nabla u\|_{\dot{B}_{2,1}^{\frac{d}{2}}}\|\tau\|_{\dot{B}_{2,1}^{\frac{d}{2}}} \leq C\left(\left\|u^{h}\right\|_{\dot{B}_{2,1}^{\frac{d}{2}+1}}+\left\|u^{d}\right\|_{\dot{B}_{2,1}^{\frac{d}{2}+1}}\right)\|\tau\|_{\dot{B}_{2,1}^{\frac{d}{2}}} .
\end{aligned}
$$

Combining the above estimates, we find that

$$
\sum_{q \in \mathbb{Z}} 2^{q\left(\frac{d}{2}-1\right)}\left\|\tilde{h}_{q}^{i}\right\|_{L^{2}} \leq C\left(\left\|u^{h}\right\|_{\dot{B}_{2,1}^{\frac{d}{2}+1}}+\left\|u^{d}\right\|_{\dot{B}_{2,1}^{\frac{d}{2}+1}}\right)\|\tau\|_{\dot{B}_{2,1}^{\frac{d}{2}}} .
$$

Substituting (3.51) and (3.55) into (3.48) yields

$$
\begin{aligned}
& \left\|u^{i}\right\|_{\dot{B}_{2,1}^{\frac{d}{2}-1}}+(1-\omega)\left\|\sigma^{i}\right\|_{\dot{B}_{2,1}^{\frac{d}{2}-1}}+(1-\omega)\left\|u^{i}\right\|_{L_{t}^{1}\left(\dot{B}_{2,1}^{\frac{d}{2}+1}\right)}+\left\|\sigma^{i}\right\|_{L_{t}^{1}\left(\dot{B}_{2,1}^{\frac{d}{2}+1, \frac{d}{2}-1}\right)} \\
& \leq \frac{C}{(1-\omega)^{2}}\left(\left\|u_{0}^{i}\right\|_{\dot{B}_{2,1}^{\frac{d}{2}-1}}+\left\|\sigma_{0}^{i}\right\|_{\dot{B}_{2,1}^{\frac{d}{2}-1}}+\int_{0}^{t}\left(\left\|u^{h}\right\|_{\dot{B}_{2,1}^{\frac{d}{2}+1}}+\left\|u^{d}\right\|_{\dot{B}_{2,1}^{\frac{d}{2}+1}}\right)\|\tau\|_{\dot{B}_{2,1}^{\frac{d}{2}}} d t^{\prime}\right. \\
& +\int_{0}^{t}\left(\left\|u^{h}\right\|_{\dot{B}_{2,1}^{\frac{d}{2}-1}}\left\|u^{h}\right\|_{\dot{B}_{2,1}^{\frac{d}{2}+1}}+\left\|u^{h}\right\|_{\dot{B}_{2,1}^{\frac{d}{2}+1}}^{\frac{1}{2}}\left\|u_{\dot{B}_{2,1}^{d} \|^{\frac{d}{2}-1}}^{\frac{1}{2}}\right\| u^{h}\left\|_{\dot{B}_{2,1}^{\frac{d}{2}-1}}^{\frac{1}{2}}\right\| u_{\dot{B}_{2,1}^{d}}^{\frac{d}{2}+1}\right) d t^{\prime} \\
& \left.+\int_{0}^{t}\left\|u^{h}\right\|_{\dot{B}_{2,1}^{\frac{d}{2}+1}}^{\frac{1}{2}}\|u\|_{\dot{B}_{2,1}^{\frac{d}{2}+1}}^{\frac{1}{2}}\left(\left\|u^{i}\right\|_{\dot{B}_{2,1}^{\frac{d}{2}-1}}+\left\|\sigma^{i}\right\|_{\dot{B}_{2,1}^{\frac{d}{2}-1}}\right) d t^{\prime}\right) \\
& \leq \frac{C}{(1-\omega)^{2}}\left(\left\|u_{0}^{i}\right\|_{\dot{B}_{2,1}^{\frac{d}{2}-1}}+\left\|\sigma_{0}^{i}\right\|_{\dot{B}_{2,1}^{\frac{d}{2}-1}}+\int_{0}^{t}\left(\left\|u^{h}\right\|_{\dot{B}_{2,1}^{\frac{d}{2}+1}}+\left\|u^{d}\right\|_{\dot{B}_{2,1}^{\frac{d}{2}+1}}\right)\|\tau\|_{\dot{B}_{2,1}^{\frac{d}{2}}} d t^{\prime}\right. \\
& +\int_{0}^{t}\left(\left\|u^{h}\right\|_{\dot{B}_{2,1}^{\frac{d}{2}-1}}\left\|u^{h}\right\|_{\dot{B}_{2,1}^{\frac{d}{2}+1}}+\left\|u^{h}\right\|_{\dot{B}_{2,1}^{\frac{d}{2}+1}}^{\frac{1}{2}}\left\|u^{d}\right\|_{\dot{B}_{2,1}^{\frac{d}{2}-1}}^{\frac{1}{2}}\left\|u^{h}\right\|_{\dot{B}_{2,1}^{\frac{d}{2}-1}}^{\frac{1}{2}} \| u_{\dot{B}_{2,1}^{d}}^{\frac{d}{2}+1}\right) d t^{\prime} \\
& \left.+\int_{0}^{t}\left(\left\|u^{h}\right\|_{\dot{B}_{2,1}^{\frac{d}{2}+1}}+\left\|u^{h}\right\|_{\dot{B}_{2,1}^{\frac{d}{2}+1}}^{\frac{1}{2}}\left\|u^{d}\right\|_{\dot{B}_{2,1}^{\frac{d}{2}+1}}^{\frac{1}{2}}\right)\left\|u^{i}\right\|_{\dot{B}_{2,1}^{\frac{d}{2}-1}} d t^{\prime}\right) \text { for all } i \in\{1,2, \cdots, d\} \text {. }
\end{aligned}
$$


Consequently, there exist two constants $C_{1}$ and $C_{2}$ depending on $d, \operatorname{Re}$ and $\mathrm{We}$, but independent of $\omega$, such that (3.3) and (3.4) hold.

\section{The damping effect of $\tau$.}

In order to deal with the nonlinear coupling between $u$ and $\tau$, it is necessary to take advantage of the damping effect of $\tau$. To do so, applying $\dot{\Delta}_{q}$ to the equation of $\tau$ yields

$$
\partial_{t} \tau_{q}+\dot{S}_{q-1} u \cdot \nabla \tau_{q}+\frac{\tau_{q}}{\mathrm{We}}=\frac{2 \omega}{\mathrm{We}} D\left(u_{q}\right)+\dot{S}_{q-1} u \cdot \nabla \tau_{q}-\dot{\Delta}_{q}(u \cdot \nabla \tau)-\dot{\Delta}_{q} g_{\alpha}(\tau, \nabla u) .
$$

Taking the inner product of the above equation with $\tau_{q}$, integrating by parts, we have

$$
\begin{aligned}
& \frac{1}{2} \frac{d}{d t}\left\|\tau_{q}\right\|_{L^{2}}^{2}+\frac{1}{\mathrm{We}}\left\|\tau_{q}\right\|_{L^{2}}^{2} \\
= & \frac{2 \omega}{\mathrm{We}}\left(D\left(u_{q}\right) \mid \tau_{q}\right)+\left(\dot{S}_{q-1} u \cdot \nabla \tau_{q}-\dot{\Delta}_{q}(u \cdot \nabla \tau) \mid \tau_{q}\right)-\left(\dot{\Delta}_{q} g_{\alpha}(\tau, \nabla u) \mid \tau_{q}\right) .
\end{aligned}
$$

It follows that

$$
\begin{aligned}
\left\|\tau_{q}(t)\right\|_{L^{2}}+\frac{1}{\mathrm{We}}\left\|\tau_{q}\right\|_{L_{t}^{1}\left(L^{2}\right)} \leq & C\left(\left\|\tau_{q}(0)\right\|_{L^{2}}+\omega 2^{q}\left\|u_{q}\right\|_{L^{2}}+\left\|\dot{S}_{q-1} u \cdot \nabla \tau_{q}-\dot{\Delta}_{q}\left(\dot{T}_{u^{k}} \partial_{k} \tau\right)\right\|_{L^{2}}\right. \\
& \left.+\left\|\dot{\Delta}_{q} \dot{T}_{\partial_{k} \tau}^{\prime} u^{k}\right\|_{L^{2}}+\left\|\dot{\Delta}_{q} g_{\alpha}(\tau, \nabla u)\right\|_{L^{2}}\right) .
\end{aligned}
$$

Multiplying the above equation by $2^{q \frac{d}{2}}$, and summing over $q \in \mathbb{Z}$, we find that there exists a constant $C_{3}$ depending on $d, \operatorname{Re}$ and We, but independent of $\omega$, such that

$$
\begin{aligned}
& \|\tau\|_{\tilde{L}_{t}^{\infty}\left(\dot{B}_{2,1}^{\frac{d}{2}}\right)}+\|\tau\|_{L_{t}^{1}\left(\dot{B}_{2,1}^{\frac{d}{2}}\right)} \\
& \leq C_{3}\left(\left\|\tau_{0}\right\|_{\dot{B}_{2,1}^{\frac{d}{2}}}+\omega\|u\|_{L_{t}^{1}\left(\dot{B}_{2,1}^{\frac{d}{2}+1}\right)}+\int_{0}^{t}\|u\|_{\dot{B}_{2,1}^{\frac{d}{2}+1}}\|\tau\|_{\dot{B}_{2,1}^{\frac{d}{2}}} d t^{\prime}\right) \\
& \leq C_{3}\left(\left\|\tau_{0}\right\|_{\dot{B}_{2,1}^{\frac{d}{2}}}+\omega\left(\left\|u^{h}\right\|_{L_{t}^{1}\left(\dot{B}_{2,1}^{\frac{d}{2}+1}\right)}+\left\|u^{d}\right\|_{L_{t}^{1}\left(\dot{B}_{2,1}^{\frac{d}{2}+1}\right)}\right)+\int_{0}^{t}\left(\left\|u^{h}\right\|_{\dot{B}_{2,1}^{\frac{d}{2}+1}}+\left\|u^{d}\right\|_{\dot{B}_{2,1}^{\frac{d}{2}+1}}\right)\|\tau\|_{\dot{B}_{2,1}^{\frac{d}{2}}} d t^{\prime}\right)
\end{aligned}
$$

where we have used the following nonlinear estimates which can be obtained in a similar manner to those of (3.52), (3.53) and (3.54),

$$
\sum_{q \in \mathbb{Z}} 2^{\frac{d}{2} q}\left\|\dot{S}_{q-1} u \cdot \nabla \tau_{q}-\dot{\Delta}_{q}\left(\dot{T}_{u^{k}} \partial_{k} \tau\right)\right\|_{L^{2}} \leq C\|\nabla u\|_{L^{\infty}\|\tau\|_{\dot{B}_{2,1}^{\frac{d}{2}}}},
$$

and

$$
\left\|\dot{T}_{\partial_{k} \tau}^{\prime} u^{k}\right\|_{\dot{B}_{2,1}^{\frac{d}{2}}} \leq C\|u\|_{\dot{B}_{2,1}^{\frac{d}{2}+1}}\|\tau\|_{\dot{B}_{2,1}^{\frac{d}{2}}}, \quad\left\|g_{\alpha}(\tau, \nabla u)\right\|_{\dot{B}_{2,1}^{\frac{d}{2}}} \leq C\|\nabla u\|_{\dot{B}_{2,1}^{\frac{d}{2}}}\|\tau\|_{\dot{B}_{2,1}^{\frac{d}{2}-1}}
$$

This completes the proof Proposition 3.1

Remark 3.1. We can bound $\sum_{q \in \mathbb{Z}} 2^{q\left(\frac{d}{2}-1\right)}\left\|\tilde{h}_{q}^{i}\right\|_{L^{2}}$ without making a distinction between horizontal direction and vertical direction due to the appearance of the nonlinear term $g_{\alpha}(\tau, \nabla u)$. Our above estimates of $\sum_{q \in \mathbb{Z}} 2^{q\left(\frac{d}{2}-1\right)}\left\|\tilde{h}_{q}^{i}\right\|_{L^{2}}$ aim to reveal that the convection term $u \cdot \nabla \tau$ behaves better than $g_{\alpha}(\tau, \nabla u)$.

The rest part of this section is to close the estimates (3.3)-(3.5) in Proposition 3.1. Before proceeding any further, let us denote

$$
\begin{aligned}
& A_{0}^{d}:=\frac{4 C_{2}}{(1-\omega)^{3}}\left(A^{d}(0)+1\right), \\
& A_{0}^{h}:=\frac{16 C_{1} C_{2} C_{3}}{(1-\omega)^{6}} \exp \left(\frac{8\left(C_{1}^{2}+C_{3}\right)}{(1-\omega)^{6}}\left(A_{0}^{d}\right)^{2}\right)\left(A^{h}(0)+\omega A^{d}(0)+B(0)\right), \\
& B_{0}:=\frac{192 C_{1} C_{2}^{2} C_{3}^{2}}{(1-\omega)^{6}} \exp \left(\frac{12\left(C_{1}^{2}+C_{3}\right)}{(1-\omega)^{6}}\left(A_{0}^{d}\right)^{2}\right)\left(A^{h}(0)+\omega A^{d}(0)+B(0)\right) .
\end{aligned}
$$


Proposition 3.2. Assume that $(u, \tau)$ is a solution of system (1.1) on $[0, T]$, satisfying

$$
A^{h}(T) \leq A_{0}^{h}, \quad A^{d}(T) \leq A_{0}^{d}, \quad B(T) \leq B_{0} .
$$

If

$$
\begin{gathered}
\frac{3}{2} A_{0}^{h}+\sqrt{A_{0}^{h} A_{0}^{d}}+B_{0} \leq \frac{(1-\omega)^{3}}{2 C_{2}}, \quad A_{0}^{h}\left(A_{0}^{h}+B_{0}\right) \leq 1, \\
B_{0}\left(1+\frac{2 C_{2}}{(1-\omega)^{3}}\left(A_{0}^{h}+B_{0}\right)\right) \leq \frac{(1-\omega)^{3}}{4 C_{1}}, \\
\frac{3}{2} A_{0}^{h} \leq 1, \quad \frac{2 C_{2} C_{3}}{(1-\omega)^{3}} A_{0}^{h} \leq \frac{1}{2},
\end{gathered}
$$

then there hold

$$
A^{h}(T) \leq \frac{1}{2} A_{0}^{h}, \quad A^{d}(T) \leq \frac{1}{2} A_{0}^{d}, \quad \text { and } \quad B(T) \leq \frac{1}{2} B_{0} .
$$

Proof. From (3.4), it is easy to verify that

$$
\begin{aligned}
& A^{d}(t) \leq \frac{C_{2}}{(1-\omega)^{3}}\left(A^{d}(0)+A^{h}(t)\left(A^{h}(t)+B(t)\right)+\frac{1}{2} A^{h}(t)\left\|u^{d}\right\|_{\tilde{L}_{t}^{\infty}\left(\dot{B}_{2,1}^{\frac{d}{2}-1}\right)}\right. \\
& \left.+\frac{1}{2} A^{h}(t)\left\|u^{d}\right\|_{L_{t}^{1}\left(\dot{B}_{2,1}^{\frac{d}{2}+1}\right)}+\left(A^{h}(t)+\sqrt{A^{h}(t) A^{d}(t)}\right)\left\|u^{d}\right\|_{\tilde{L}_{t}^{\infty}\left(\dot{B}_{2,1}^{2}\right)}+B(t)\left\|u^{d}\right\|_{L_{t}^{1}\left(\dot{B}_{2,1}^{2}+1\right.}^{\frac{d}{2}+1}\right) \\
& \leq \frac{C_{2}}{(1-\omega)^{3}}\left(A^{d}(0)+A^{h}(t)\left(A^{h}(t)+B(t)\right)+\left(\frac{3}{2} A^{h}(t)+\sqrt{A^{h}(t) A^{d}(t)}+B(t)\right) A^{d}(t)\right) \text {. }
\end{aligned}
$$

If

$$
\frac{3}{2} A_{0}^{h}+\sqrt{A_{0}^{h} A_{0}^{d}}+B_{0} \leq \frac{(1-\omega)^{3}}{2 C_{2}},
$$

then it follows from (3.57) and (3.62) that

$$
A^{d}(t) \leq \frac{2 C_{2}}{(1-\omega)^{3}}\left(A^{d}(0)+A^{h}(t)\left(A^{h}(t)+B(t)\right)\right), \quad t \in[0, T] .
$$

Furthermore, if

$$
A_{0}^{h}\left(A_{0}^{h}+B_{0}\right) \leq 1
$$

then (3.64) changes to be

$$
A^{d}(t) \leq \frac{2 C_{2}}{(1-\omega)^{3}}\left(A^{d}(0)+1\right)=\frac{1}{2} A_{0}^{d}, \quad t \in[0, T] .
$$

Next we go to bound $B(t)$. To do this, combining (3.56) and (3.64), we are led to

$$
\begin{aligned}
B(t) \leq & C_{3}\left(B(0)+\omega A_{0}^{h}+\omega A^{d}(t)+\int_{0}^{t}\left(\left\|u^{h}\right\|_{\dot{B}_{2,1}^{\frac{d}{2}+1}}+\left\|u^{d}\right\|_{\dot{B}_{2,1}^{\frac{d}{2}+1}}\right)\|\tau\|_{\dot{B}_{2,1}^{\frac{d}{2}}} d t^{\prime}\right) \\
\leq & C_{3}\left(B(0)+\omega A_{0}^{h}+\frac{2 C_{2} \omega}{(1-\omega)^{3}}\left(A^{d}(0)+\left(A^{h}(t)\right)^{2}+A^{h}(t) B(t)\right)\right. \\
& \left.+\int_{0}^{t}\left(\left\|u^{h}\right\|_{\dot{B}_{2,1}^{\frac{d}{2}+1}}+\left\|u^{d}\right\|_{\dot{B}_{2,1}^{\frac{d}{2}+1}}\right)\|\tau\|_{\dot{B}_{2,1}^{\frac{d}{2}}} d t^{\prime}\right) \\
\leq & C_{3}\left(B(0)+\frac{3}{2} \omega A_{0}^{h}+\frac{2 C_{2} \omega}{(1-\omega)^{3}} A^{d}(0)+\int_{0}^{t}\left(\left\|u^{h}\right\|_{\dot{B}_{2,1}^{\frac{d}{2}+1}}+\left\|u^{d}\right\|_{\dot{B}_{2,1}^{\frac{d}{2}+1}}\right)\|\tau\|_{\dot{B}_{2,1}^{\frac{d}{2}}} d t^{\prime}\right)+\frac{1}{2} B(t),
\end{aligned}
$$

provided

$$
\frac{2 C_{2} C_{3}}{(1-\omega)^{3}} A_{0}^{h} \leq \frac{1}{2}
$$


Using Gronwall's inequality, we obtain

$$
B(t) \leq 2 C_{3} \exp \left(2 C_{3}\left(1+A_{0}^{d}\right)\right)\left(B(0)+\frac{3}{2} \omega A_{0}^{h}+\frac{2 C_{2} \omega}{(1-\omega)^{3}} A^{d}(0)\right) \leq \tilde{B}_{0},
$$

where $\tilde{B}_{0}$ is defined to be

$$
\tilde{B}_{0}:=2 C_{3} \exp \left(2 C_{3}\left(1+A_{0}^{d}\right)\right)\left(B(0)+\frac{3}{2} \omega+\frac{2 C_{2} \omega}{(1-\omega)^{3}} A^{d}(0)\right) .
$$

To estimate $A^{h}(t)$, we infer from (3.3) that

$$
\begin{aligned}
A^{h}(t) \leq & \frac{C_{1}}{(1-\omega)^{3}}\left(A^{h}(0)+\int_{0}^{t}\left(\left\|u^{h}\right\|_{\dot{B}_{2,1}^{\frac{d}{2}+1}}+\frac{C_{1}}{(1-\omega)^{3}}\left\|u^{d}\right\|_{\dot{B}_{2,1}^{\frac{d}{2}-1}}\left\|u^{d}\right\|_{\dot{B}_{2,1}^{\frac{d}{2}+1}}\right)\left\|u^{h}\right\|_{\dot{B}_{2,1}^{\frac{d}{2}-1}} d t^{\prime}\right. \\
& \left.+\frac{(1-\omega)^{3}}{4 C_{1}}\left\|u^{h}\right\|_{L_{t}^{1}\left(\dot{B}_{2,1}^{\frac{d}{2}+1}\right)}+\frac{1}{2} \int_{0}^{t}\left\|u^{h}\right\|_{\dot{B}_{2,1}^{\frac{d}{2}+1}}+\left\|u^{d}\right\|_{\dot{B}_{2,1}^{\frac{d}{2}+1}}\right)\left\|u^{h}\right\|_{\dot{B}_{2,1}^{\frac{d}{2}-1}} d t^{\prime} \\
& \left.+\|\tau\|_{L_{t}^{\infty}\left(\dot{B}_{2,1}^{\frac{d}{2}}\right)}\left\|u^{h}\right\|_{L_{t}^{1}\left(\dot{B}_{2,1}^{\frac{d}{2}+1}\right)}+\int_{0}^{t}\left\|u^{d}\right\|_{\dot{B}_{2,1}^{\frac{d}{2}+1}}\|\tau\|_{\dot{B}_{2,1}^{\frac{d}{2}}} d t^{\prime}\right) \\
\leq & \frac{C_{1}}{(1-\omega)^{3}}\left(A^{h}(0)+\|\tau\|_{L_{t}^{\infty}\left(\dot{B}_{2,1}^{\frac{d}{2}}\right)}\left\|u^{d}\right\|_{L_{t}^{1}\left(\dot{B}_{2,1}^{\frac{d}{2}+1}\right)}+\left(\frac{(1-\omega)^{3}}{4 C_{1}}+B(t)\right)\left\|u^{h}\right\|_{L_{t}^{1}\left(\dot{B}_{2,1}^{\frac{d}{2}+1}\right)}\right. \\
& \left.+\int_{0}^{t}\left(\frac{3}{2}\left\|u^{h}\right\|_{\dot{B}_{2,1}^{\frac{d}{2}+1}}+\left(\frac{C_{1}}{(1-\omega)^{3}} A_{0}^{d}+\frac{1}{2}\right)\left\|u^{d}\right\|_{\dot{B}_{2,1}^{\frac{d}{2}+1}}\right)\left\|u^{h}\right\|_{\dot{B}_{2,1}^{\frac{d}{2}-1}} d t^{\prime}\right) .
\end{aligned}
$$

In view of (3.64), we have

$$
\begin{aligned}
\|\tau\|_{L_{t}^{\infty}\left(\dot{B}_{2,1}^{\frac{d}{2}}\right)}\left\|u^{d}\right\|_{L_{t}^{1}\left(\dot{B}_{2,1}^{\frac{d}{2}}\right)} & \leq \frac{2 C_{2}}{(1-\omega)^{3}}\left(A^{d}(0)+A^{h}(t)\left(A^{h}(t)+B(t)\right)\right) B(t) \\
& \leq \frac{2 C_{2}}{(1-\omega)^{3}} A^{d}(0) B(t)+\frac{2 C_{2}}{(1-\omega)^{3}}\left(A^{h}(t)+B(t)\right) B(t) A^{h}(t) .
\end{aligned}
$$

Substituting (3.72) into (3.71), and using (3.69), one deduces that

$$
\begin{aligned}
A^{h}(t) \leq & \frac{C_{1}}{(1-\omega)^{3}}\left(A^{h}(0)+\frac{2 C_{2}}{(1-\omega)^{3}} A^{d}(0) \tilde{B}_{0}+\left(B(t)\left(1+\frac{2 C_{2}}{(1-\omega)^{3}}\left(A^{h}(t)+B(t)\right)\right)\right) A^{h}(t)\right. \\
& \left.+\int_{0}^{t}\left(\frac{3}{2}\left\|u^{h}\right\|_{\dot{B}_{2,1}^{\frac{d}{2}+1}}+\left(\frac{C_{1}}{(1-\omega)^{3}} A_{0}^{d}+\frac{1}{2}\right)\left\|u^{d}\right\|_{\dot{B}_{2,1}^{\frac{d}{2}+1}}\right)\left\|u^{h}\right\|_{\dot{B}_{2,1}^{\frac{d}{2}-1}} d t^{\prime}\right)+\frac{1}{4} A^{h}(t) .
\end{aligned}
$$

If

$$
B_{0}\left(1+\frac{2 C_{2}}{(1-\omega)^{3}}\left(A_{0}^{h}+B_{0}\right)\right) \leq \frac{(1-\omega)^{3}}{4 C_{1}}
$$

then (3.73) reduces to

$$
\begin{aligned}
A^{h}(t) \leq & \frac{2 C_{1}}{(1-\omega)^{3}}\left(A^{h}(0)+\frac{2 C_{2}}{(1-\omega)^{3}} A^{d}(0) \tilde{B}_{0}\right. \\
& \left.+\int_{0}^{t}\left(\frac{3}{2}\left\|u^{h}\right\|_{\dot{B}_{2,1}^{\frac{d}{2}+1}}+\left(\frac{C_{1}}{(1-\omega)^{3}} A_{0}^{d}+\frac{1}{2}\right)\left\|u^{d}\right\|_{\dot{B}_{2,1}^{\frac{d}{2}+1}}\right)\left\|u^{h}\right\|_{\dot{B}_{2,1}^{\frac{d}{2}-1}} d t^{\prime}\right) .
\end{aligned}
$$

Gronwalll's inequality implies that

$$
A^{h}(t) \leq \frac{2 C_{1}}{(1-\omega)^{3}} \exp \left(\frac{2 C_{1}}{(1-\omega)^{3}}\left(1+A_{0}^{d}\left(1+\frac{C_{1}}{(1-\omega)^{3}} A_{0}^{d}\right)\right)\right)\left(A^{h}(0)+\frac{2 C_{2}}{(1-\omega)^{3}} A^{d}(0) \tilde{B}_{0}\right),
$$

provided

$$
\frac{3}{2} A_{0}^{h} \leq 1
$$


Substituting (3.70) into (3.76), we find that

$$
\begin{aligned}
A^{h}(t) \leq & \frac{2 C_{1}}{(1-\omega)^{3}} \exp \left(\frac{2 C_{1}^{2}}{(1-\omega)^{6}}\left(1+A_{0}^{d}\right)^{2}\right)\left\{A^{h}(0)\right. \\
& \left.+\frac{4 C_{2} C_{3}}{(1-\omega)^{3}} \exp \left(2 C_{3}\left(1+A_{0}^{d}\right)\right)\left(A^{d}(0) B(0)+\omega A^{d}(0) A_{0}^{d}\right)\right\} \\
\leq & \frac{2 C_{1}}{(1-\omega)^{3}} \exp \left(\frac{8 C_{1}^{2}}{(1-\omega)^{6}}\left(A_{0}^{d}\right)^{2}\right)\left\{A^{h}(0)+\frac{4 C_{2} C_{3}}{(1-\omega)^{3}} \exp \left(4 C_{3} A_{0}^{d}\right) A_{0}^{d}\left(B(0)+\omega A^{d}(0)\right)\right\} \\
\leq & \frac{8 C_{1} C_{2} C_{3}}{(1-\omega)^{6}} \exp \left(\frac{8\left(C_{1}^{2}+C_{3}\right)}{(1-\omega)^{6}}\left(A_{0}^{d}\right)^{2}\right)\left(A^{h}(0)+\omega A^{d}(0)+B(0)\right)=\frac{1}{2} A_{0}^{h} .
\end{aligned}
$$

Finally, substituting (3.78) into (3.69), we get the estimate of $B(t)$ in term of $A^{h}(0)+\omega A^{d}(0)+B(0)$ :

$$
\begin{aligned}
B(t) & \leq \frac{48 C_{1} C_{2} C_{3}^{2}}{(1-\omega)^{6}} \exp \left(4 C_{3} A_{0}^{d}\right) \exp \left(\frac{8\left(C_{1}^{2}+C_{3}\right)}{(1-\omega)^{6}}\left(A_{0}^{d}\right)^{2}\right)\left(2 B(0)+A^{h}(0)+2 \omega A^{d}(0)\right) \\
& \leq \frac{96 C_{1} C_{2}^{2} C_{3}^{2}}{(1-\omega)^{6}} \exp \left(\frac{12\left(C_{1}^{2}+C_{3}\right)}{(1-\omega)^{6}}\left(A_{0}^{d}\right)^{2}\right)\left(A^{h}(0)+\omega A^{d}(0)+B(0)\right)=\frac{1}{2} B_{0}
\end{aligned}
$$

The proof of Proposition 3.2 is completed.

\section{Proof of Theorem1.1}

Now we are in a position to prove the maim result of this paper.

Proof. The local existence and uniqueness of the solution $(u, \tau) \in \mathcal{E}_{T}^{\frac{d}{2}}$ to system (1.1) with initial data $\left(u_{0}, \tau_{0}\right) \in\left(\dot{B}_{2,1}^{\frac{d}{2}-1}\right)^{d} \times\left(\dot{B}_{2,1}^{\frac{d}{2}}\right)^{d \times d}$ have been proved by Chemin and Masmoudi [7]. Let $T^{\star}$ be the lifespan of $(u, \tau)$ obtained in [7]. Define $T_{1}$ be the supremum of all time $T^{\prime} \in\left[0, T^{*}\right)$ such that

$$
A^{h}(t) \leq A_{0}^{h}, \quad A^{d}(t) \leq A_{0}^{d}, \quad B(t) \leq B_{0} \quad \text { for all } t \in\left[0, T^{\prime}\right] .
$$

Choosing $C_{0}$ satisfying (1.8) so large that (3.58)-(3.60) hold, then thanks to Proposition 3.2, for all $0 \leq t<T_{1}$, we have

$$
A^{h}(t) \leq \frac{1}{2} A_{0}^{h}, \quad A^{d}(t) \leq \frac{1}{2} A_{0}^{d}, \quad B(t) \leq \frac{1}{2} B_{0} .
$$

According to standard continuation method, we obtain $T_{1}=T^{\star}$. Moreover, (4.2) and the embedding $\dot{B}_{2,1}^{\frac{d}{2}} \hookrightarrow L^{\infty}$ imply that

$$
\int_{0}^{T^{\star}}\left(\|\nabla u(t, \cdot)\|_{L^{\infty}}+\|\tau(t, \cdot)\|_{L^{\infty}}\right) d t<\infty
$$

provided (1.8) holds. Thus, the blow-up criterion in [7] enables us to conclude that $T^{\star}=\infty$. This completes the proof of Theorem 1.1 .

\section{Acknowledgments}

Research supported by China Postdoctoral Science Foundation funded project 2014M552065, and National Natural Science Foundation of China 11401237, 11271322, and 11331005. 


\section{REFERENCES}

[1] H. Bahouri, J.-Y. Chemin and R. Danchin, "Fourier analysis and nonlinear partial differential equations". Grundlehren der Mathematischen Wissenschaften [Fundamental Principles of Mathematical Sciences], 343. Springer, Heidelberg, 2011.

[2] J.-M. Bony, Calcul symbolique et propagation des singularités pour équations aux dérivées partielles nonlinéaires. Annales Scinentifiques de l'école Normale Supérieure 14(1981), 209-246.

[3] J.-Y. Chemin, "Localization in Fourier space and Navier-Stokes system, Phase Space Analysis of Partial Differential Equations". Proceedings 2004, CRM series, Pisa 2004, 53-136.

[4] J.-Y. Chemin, N. Lerner, Flot de champs de vecteurs non Lipschitziens et équations de Navier-Stokes. J. Differential Equations 121(1995), 314-228.

[5] J.-Y. Chemin, I. Gallagher, Large, global solutions to the Navier-Stokes equations slowly varying in one direction, Trans. Amer. Math. Soc., 362(2010), 2859-2873.

[6] J.Y. Chemin, I. Gallagher, M. Paicu, Global regularity for some classes of large solutions to the Navier-Stokes equations, Ann. of Math., 173 (2011), 983-1012.

[7] J. Y. Chemin, N. Masmoudi, About lifespan of regular solutions of equations related to viscoelastic fluids. SIAM J. Math. Anal, 33(2001), 84-112.

[8] Q. L. Chen, C. X. Miao, Global well-posedness of viscoelastic fluids of Oldroyd type in Besov spaces, Nonlinear Analysis, 68(2008), 1928-1939.

[9] P. Constantin, M. Kliegl, Note on global regularity for two-dimensional Oldroyd-B fluids with diffusive stress. Arch. Rational Mech. Anal., 206(2012), 725-740.

[10] R. Danchin, Global existence in critical spaces for compressible Navier-Stokes equations. Invent. Math., 141(2000), 579-614.

[11] D.Y. Fang, M. Hieber and R. Z. Zi, Global existence results for Oldroyd-B Fluids in exterior domains: The case of non-small coupling parameters, Math. Ann., 357(2013), 687-709.

[12] D. Y. Fang, R. Z. Zi, Incompressible limit of Oldroyd-B fluids in the whole space, J. Differential Equations, 256 (2014), 2559-2602.

[13] E. Fernández-Cara, F. Guillén, R. Ortega, Some theoretical results concerning non-Newtonian fluids of the Oldroyd kind. Ann. Scuola Norm. Sup. Pisa, 26(1998), 1-29.

[14] H. Fujita and T. Kato, On the Navier-Stokes initial value problem I, Arch. Ration. Mech. Anal., 16(1964), $269-315$.

[15] C. Guillopé, J. C. Saut, Existence results for the flow of viscoelastic fluids with a differential constitutive law. Nonlinear Anal. 15(1990), 849-869.

[16] M. Hieber, Y. Naito and Y. Shibata, Global existence results for Oldroyd-B fluids in exterior domains. J. Differential Equations, 252 (2012) 2617-2629.

[17] X. P. Hu, F. H. Lin, Global solutions of two dimensional incompressible viscoelastic flows with discontinuous initial data, arXiv:1312.6749 [math.AP].

[18] R. Kupferman, C. Mangoubi, and E. S. Titi, A Beale-Kato-Majda breakdown criterion for an Oldroyd-B fluid in the creeping flow regime. Commun. Math. Sci., 6(2008), 235-256.

[19] Z. Lei, On 2D viscoelasticity with small strain, Arch. Ration. Mech. Anal., 198(2010), 13-37.

[20] Z. Lei, C. Liu and Y. Zhou, Global existence for a 2D incompressible viscoelastic model with small strain.Commun. Math. Sci., 5(2007), 595-616.

[21] Z. Lei, C. Liu and Y. Zhou, Global solutions for incompressible viscoelastic fluids, Arch. Ration. Mech. Anal., 188(2008), 371-398.

[22] Z. Lei, N. Masmoudi, Y. Zhou, Remarks on the blowup criteria for Oldroyd models, J. Differential Equations, 248(2010), 328-341.

[23] Z. Lei, Y. Zhou, Global existence of classical solutions for the two-dimensional Oldroyd model via the incompressible limit, SIAM J. Math. Anal., 37(2005), 797-814.

[24] F. H. Lin, C. Liu and P. Zhang, On hydrodynamics of viscoelastic fluids, Comm. Pure Appl. Math., 58(2005), 14371471.

[25] F. H. Lin, P. Zhang, On the initial-boundary value problem of the incompressible viscoelastic fluid system, Comm. Pure Appl. Math., 61(2008), 539-558.

[26] P. L. Lions, N. Masmoudi, Global solutions for some Oldroyd models of non-Newtonian flows. Chinese Ann. Math. Ser. B, 21(2000), 131-146.

[27] L. Molinet, R. Talhouk, On the global and periodic regular flows of viscoelastic fluids with a differential constitutive law. Nonlinear Diff. Equations Appl. 11(2004), 349-359.

[28] J. G. Oldroyd, Non-Newtonian effects in steady motion of some idealized elastico-viscous liquids. Proc. Roy. Soc. London., 245(1958), 278-297.

[29] M. Paicu, P. Zhang, Global solutions to the 3-D incompressible anisotropic Navier-Stokes system in the critical spaces, Comm. Math. Phys., 307(2011), 713-759.

[30] J. Z. Qian, Well-posedness in critical spaces for incompressible viscoelastic fluid system, Nonlinear Analysis, 72(2010), 3222-3234. 
[31] R. Talhouk , “Analyse Mathématique de Quelques Écoulements de Fluides Viscoélastiques,” Thèse, Université ParisSud, 1994.

[32] T. Zhang, D. Y. Fang, Global existence of strong solution for equations related to the incompressible viscoelastic fluids in the critical $L^{p}$ framework, SIAM J. Math. Anal., 44(2012), 2266-2288.

[33] R. Z. Zi, D. Y. Fang and T. Zhang, Global solution to the incompressible Oldroyd-B model in the critical $L^{p}$ framework: the case of the non-small coupling parameter, Arch. Rational Mech. Anal., 213(2014), 651-687.

[34] R. Z. Zi, Global solution to the incompressible Oldroyd-B model in hybrid Besov spaces, arXiv:1410.7486 [math.AP].

[35] R. Z. Zi, Global solution in critical spaces to the compressible Oldroyd-B model with non-small coupling parameter, arXiv:1410.7483 [math.AP].

[36] T. Zhang, Global wellposedness prolem for the 3-D incompressible anisotropic Navier-Stokes equa- tions in an anisotropic space, Comm. Math. Phys., 287(2009), 211-224.

[37] T. Zhang, Global strong solutions for equations related to the incompressible viscoelastic fluids with a class of large initial data, Nonlinear Analysis, 100(2014), 59-77.

Department of Mathematics, Zhejiang University, Hangzhou 310027, P. R. China

E-mail address: dyf@zju.edu.cn

School of Mathematics and Statistics, Central China Normal University, Wuhan 430079, P. R. China

E-mail address: rzz@mail.ccnu.edu.cn 